\title{
Liquid Biopsy-Based Exo-oncomiRNAs Can Predict Prostate Cancer Aggressiveness
}

\author{
Xavier Ruiz-Plazas ${ }^{1,2, \dagger}$, Antonio Altuna-Coy ${ }^{1, \dagger}$, Marta Alves-Santiago ${ }^{1,2}$, José Vila-Barja ${ }^{2, \ddagger}$, \\ Joan Francesc García-Fontgivell ${ }^{1,3}$, Salomé Martínez-González ${ }^{3}$, José Segarra-Tomás ${ }^{1,2, *, \S}$ \\ and Matilde R. Chacón ${ }^{1, *, \&[}$
}

Citation: Ruiz-Plazas, X.;

Altuna-Coy, A.; Alves-Santiago, M. Vila-Barja, J.; García-Fontgivell, J.F.; Martínez-González, S.;

Segarra-Tomás, J.; Chacón, M.R.

Liquid Biopsy-Based

Exo-oncomiRNAs Can Predict Prostate Cancer Aggressiveness. Cancers 2021, 13, 250.

https://doi.org/10.3390/cancers 13020250

Received: 18 November 2020 Accepted: 7 January 2021

Published: 11 January 2021

Publisher's Note: MDPI stays neutral with regard to jurisdictional clai$\mathrm{ms}$ in published maps and institutional affiliations.

Copyright: (C) 2021 by the authors. Licensee MDPI, Basel, Switzerland. This article is an open access article distributed under the terms and conditions of the Creative Commons Attribution (CC BY) license (https:// creativecommons.org/licenses/by/ $4.0 /)$.
1 Disease Biomarkers and Molecular Mechanisms Group, IISPV, Joan XXIII University Hospital, Universitat Rovira i Virgili, 43007 Tarragona, Spain; xarupl@gmail.com (X.R.-P.); antonio.altuna@iispv.cat (A.A.-C.); martalves@hotmail.es (M.A.-S.); fontgi@yahoo.es (J.F.G.-F.)

2 Urology Unit, Joan XXIII University Hospital, 43007 Tarragona, Spain; jose.vila@urv.cat

3 Pathology Unit, Joan XXIII University Hospital, 43007 Tarragona, Spain; mgonzalez.hj23.ics@gencat.cat

* Correspondence: jsegarra.hj23.ics@gencat.cat (J.S.-T.); mrch2424@gmail.com (M.R.C.); Tel.: +34-977295500 (ext. 3406) (J.S.-T. \& M.R.C.)

+ These authors contributed equally to this work.

$\ddagger \quad$ This study is dedicated to the memory of José Vila-Barja, who passed away on 20 February 2020.

$\S$ These authors are joint senior authors on this work.

Simple Summary: The main problem encountered in the management of prostate cancer $(\mathrm{PCa})$ is the inability to distinguish slow-growing indolent tumors from aggressive tumors. It is therefore important to explore non-invasive assays for the early detection of this aggressive subtype, when it can still be treated effectively. The presence of the TWEAK cytokine in biofluids of the PCa microenvironment might drive the secretion of extracellular vesicles (EVs) containing exo-oncomicroRNAs capable of modifying the tumor microenvironment. These exo-oncomicroRNAs are potentially useful as PCa biomarkers. We identified 2 exo-oncomiRNAs isolated from semen EVs by the action of TWEAK in the tumor microenvironment and, we determined their usefulness as biomarkers of PCa prognostic. We also established, for the first time, that TWEAK modulates potential exo-oncomiRNA targets, both tightly linked to cancer progression. In conclusion, our study shows that semen detection of TWEAK-regulated exo-oncomiRNAs can improve PCa prognosis, opening new avenues for diagnosis and treatment.

Abstract: Liquid biopsy-based biomarkers, including microRNAs packaged within extracellular vesicles, are promising tools for patient management. The cytokine tumor necrosis factor-like weak inducer of apoptosis (TWEAK) is related to PCa progression and is found in the semen of patients with PCa. TWEAK can induce the transfer of exo-oncomiRNAs from tumor cells to body fluids, and this process might have utility in non-invasive PCa prognosis. We investigated TWEAK-regulated exo-microRNAs in semen and in post-digital rectal examination urine from patients with different degrees of PCa aggressiveness. We first identified 14 exo-oncomiRNAs regulated by TWEAK in PCa cells in vitro, and subsequently validated those using liquid biopsies from 97 patients with PCa. Exo-oncomiR-221-3p, -222-3p and -31-5p were significantly higher in the semen of high-risk patients than in low-risk peers, whereas exo-oncomiR-193-3p and -423-5p were significantly lower in paired samples of post-digital rectal examination urine. A panel of semen biomarkers comprising exooncomiR-221-3p, -222-3p and TWEAK was designed that could correctly classify $87.5 \%$ of patients with aggressive PCa, with $85.7 \%$ specificity and $76.9 \%$ sensitivity with an area under the curve of 0.857. We additionally found that TWEAK modulated two exo-oncomiR-221-3p targets, TCF12 and NLK. Overall, we show that liquid biopsy detection of TWEAK-regulated exo-oncomiRNAs can improve PCa prognosis prediction.

Keywords: exosomes; prostate cancer; exo-oncomiRNAS; TWEAK; semen 


\section{Introduction}

Prostate cancer (PCa) is the most commonly diagnosed cancer and the fifth leading cause of cancer-related death in men in the developed world [1]. The incidence and morbidity of PCa continues to increase, likely due to changes in eating habits and the aging of the population [2]. A major challenge in the management of PCa is the inability to distinguish slow-growing and indolent tumors from aggressive tumors, which can lead to under-treatment of patients with aggressive tumors and over-treatment of those with indolent tumors. The prostate-specific antigen (PSA) test together with the tumornodes-metastasis (TNM) stage and the Gleason score of prostate biopsy [3] are considered indisputable prognostic factors to guide treatment decision-making. Among them, only PSA is objective, making it the most extensively studied biomarker in PCa [4]. However, its lack of specificity for clinically significant tumors has led to a rise in the number of prostate biopsies performed, with a consequent increase in the diagnosis of insignificant tumors and over-treatment of patients. Accordingly, the establishment of predictive biomarkers that can distinguish between aggressive and indolent PCa would be highly valuable in clinical practice, and could reduce the risk of over-diagnosis/over-treatment. In the context of biomarker discovery, liquid biopsy has proved to be a promising non-invasive modality for cancer diagnosis and prognosis that enables the assessment of circulating molecules in biological fluids, including serum, urine and semen [5].

Inflammation predisposes to the development of cancer and promotes all stages of tumorigenesis [6]. Inflammatory molecules-including cytokines and growth factorsreleased by immune cells of the inflammatory tumor microenvironment can have a direct effect on pre-malignant and cancer cells by enhancing their proliferation and resistance to cell death and environmental stress, thereby directly promoting tumor growth and progression [6]. Tumor necrosis factor-like weak inducer of apoptosis (TWEAK) is an inflammatory cytokine that governs tumor growth by promoting inflammation and inducing angiogenesis [7], and is produced by several cells of the immune system (natural killer cells and macrophages, among others) [8]. TWEAK can typically be found as a membrane-anchored (mTWEAK) protein on the surface of cells, but it can also be released as a soluble form (sTWEAK) by proteolytic processing. Both forms function through binding to their bona fide receptor Fn14 [7], forming a receptor-ligand pair. The role of the TWEAK/Fn14 axis has been established in some solid cancers, including breast and brain cancer [7]. We have demonstrated that low serum levels of sTWEAK in head and neck cancer are related to low survival rates, a finding that we later confirmed in a large cohort of patients, overall pointing to sTWEAK as a robust non-invasive biomarker of this disease $[9,10]$. We have also established a non-invasive biomarker panel with high negative predictive value to classify PCa aggressiveness that included sTWEAK levels and Fn14 mRNA expression [11].

The release of extracellular vesicles (EVs) from cells is an active process and has been shown to be a mechanism of cell-to-cell communication [12]. Exosomes are small (nanometer-size) extracellular cargo vesicles that are secreted after the fusion of endosomes with a plasma membrane, and are released by all cell types including cancer cells $[13,14]$. Exosomes can induce functional changes to receiving cells in the premetastatic nichea specialized tumor microenvironment-for instance, aiding PCa cells to overcome the low-androgen conditions in distant metastatic organs [15]. Exosome secretion has long been linked to inflammation [16] and several experimental models have been employed to characterize the role of EVs in the development and progression of inflammatory diseases. The presence of sTWEAK in PCa tumors can not only contribute to fuel tumor progression $[17,18]$, but might also promote the secretion of EVs, which will likely have an impact on the premetastatic niche, favoring the process of migration and proliferation. This is the case for exosomes derived from TWEAK-stimulated macrophages in epithelial ovarian cancer, which have been demonstrated to be internalized by the cancer cells and inhibit cell metastasis [19].

Oncogenic shuttle miRNAs (exo-oncomiRNAs), which show long-term stability in circulation and other body fluids, have been identified in exosomes [20]. Liquid biopsy exo- 
oncomiRNAs are thus potentially informative diagnostic and / or prognostic biomarkers and might also be helpful in understanding how tumor cells transfer their oncogenic potential to the environment [21]. Several studies have demonstrated that exomiRNAs isolated from liquid biopsy might be useful for the diagnostic and risk classification of PCa $[22,23]$. In this context, the most consistently reported deregulated exomiRNAs identified as promising PCa diagnostic biomarkers in both urine and blood are miR-141, miR-375, miR-21 and Let-7 [24-27]. While miR-141 is also frequently identified to be useful for risk classification in serum [27], other exomiRNAs have been proposed as having prognostic potential such as the combination of miR-1290 and miR-375 in plasma [24] and miR-2909 in urine [28]. The literature is more scarce surrounding semen, and only miRNA-342-3p and miRNA-374b-5p have been proposed as candidates for prognosis, and miRNA-142-3p and miRNA-142-5p were described as having diagnostic potential [29]. Overall, more extensive cohort studies are needed (especially using semen) to validate the identified exomiRNAs.

Exo-oncomiRNAs can be useful tools for non-invasive diagnosis and therapy monitoring in cancer; therefore, in the present study we sought to investigate whether exooncomiRNAs are shuttled into biofluids by the action of sTWEAK in the tumor microenvironment, and to determine their usefulness as prognostic PCa biomarkers in two different liquid biopsies: semen and post-digital rectal examination urine. We also aimed to examine the downstream targets of exo-oncomiRNAs, which might be important for the control of PCa.

\section{Results}

2.1. Extracellular Vesicle-Derived Exo-oncomiRNAs Are Differentially Expressed in Liquid Biopsies from Patients with Prostate Cancer Based on the Degree of Cancer Aggressiveness

We sought to search for a useful and practicable biomarker panel capable of differentiating aggressive from non-aggressive forms of PCa in liquid biopsy-based exooncomiRNAs isolated from EVs and secreted under sTWEAK stimulating conditions. The search was divided into two phases: the initial phase was established to isolate the EV-cargo (exo-oncomiRNAs) secreted into cell culture medium of two PCa cell lines-PC-3 and $\mathrm{LNCaP}$ - treated or not with sTWEAK; in the second phase, we assayed for expression using a real-time PCR array of 752 miRNA target onco-miRNAs. We specifically chose an androgen-independent line (PC-3) and an androgen-dependent line (LNCaP). Although the two cell lines do not cover the entire spectrum of $\mathrm{PCa}$, they allowed us to implement a first approach to identify possible exo-miRNAs expressed through the influence of TWEAK [30].

Isolated EVs were confirmed by transmission electron microscopy (TEM) and by Western blot analysis of selected EV markers in order to comply with the guidelines of the International Society of Extracellular Vesicles [31]. Results confirmed the presence of EVs within the expected range (30-100 nm), which were enriched for CD9, CD63 and CD81 markers (Figure 1). The detailed results of immunoblotting are shown in Figure S1.

By screening a 752-miRNA panel, the following 14 exo-oncomiRNAs were selected from the first phase study that were significantly altered after sTWEAK treatment, comparing either PC-3 or/and LNCaP cell lines, which accomplished the following criteria: cycle threshold $(\mathrm{Ct})<33$ and at least $>1.8$-fold-over-expression when comparing both sTWEAKstimulated cell lines (Table S1): miR-125b-1-3p, miR-193b-3p, miR-221-3p, miR-222-3p, miR-23a-3p, miR-27a-3p, miR-29a-3p, miR-31-5p, miR-497-5p, miR-643, miR-663b, miR-940, miR-9-5p and miR-99a-3p.

In the second phase of the experimental approach, we evaluated the expression levels of the 14 selected exo-oncomiRNAs in EVs isolated from liquid biopsy (semen and postdigital rectal examination urine) from 97 patients with low- or high-risk PCa. Pathological and clinical characteristics of patients are listed in Table 1. Gleason grade (GG) criteria and TNM classification was determined in accordance with the International Society of Urological Pathology (ISUP). Complementary examinations included prostate volume, measured by transrectal ultrasound, and PSA, as in standard clinical practice. 
(a)

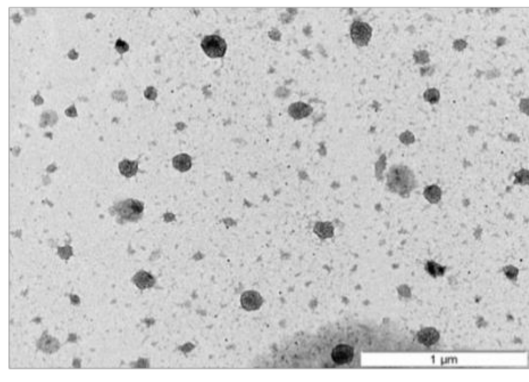

$40 \mathrm{k}$

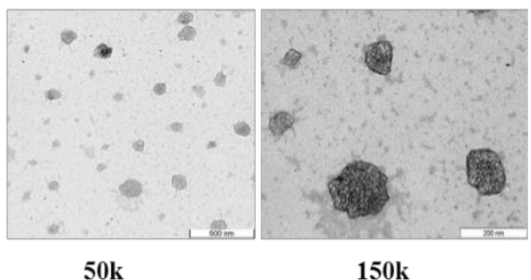

(b)



Figure 1. Characterization of isolated extracellular vesicles. (a) Analysis of extracellular vesicles (EVs) by electron microscopy at different magnification. (b) Western blot image of protein extracts prepared from EVs isolated from PC-3 culture media $(\mathrm{CM})$, post-digital rectal examination urine (U), semen (S) and total cell extract from PC-3 (C), and tested with the following antibodies: CD9, CD81, CD63, TSG101, HSP70 and tubulin. Uncropped Western Blot image is available in Figure S1.

Analysis of the expression pattern of the 14 selected exo-oncomiRNAs in liquid biopsy of semen and post-digital rectal examination urine from patients with high-risk (ISUP Group III, IV and V) and low-risk (ISUP Group I and II) PCa revealed significant differences in the following five exo-oncomiRNAs: exo-oncomiR-221-3p, exo-oncomiR-222$3 p$, exo-oncomiR-31-5p, which were up-regulated in semen of high-risk patients versus low-risk patients; and exo-oncomiR-193-3p and exo-oncomiR-423-5p, which were downregulated in post-digital rectal examination urine samples of high-risk patients (Figure 2). There were no significant differences between the studied groups for the remaining nine exo-oncomiRNAs (Table S2).

Table 1. Clinical and pathological characteristics of the studied cohort.

\begin{tabular}{|c|c|c|}
\hline Patient's Characteristics & Mean \pm SD & $N$ \\
\hline Age (years) & $63.5 \pm 6.35$ & 97 \\
\hline Prostatic Volume (c.c) & $47.49 \pm 23.09$ & 97 \\
\hline Testosterone (nmol/L) & $14.37 \pm 5.07$ & 97 \\
\hline \multirow[t]{2}{*}{ Total PSA (ng/mL) } & $9.57 \pm 7.92$ & 97 \\
\hline & & $N(\%)$ \\
\hline BMI $\left(\mathrm{kg} / \mathrm{m}^{2}\right)$ & $\begin{aligned} &<25 \\
& 25 \leq x \leq 29.99 \\
& \geq 30\end{aligned}$ & $\begin{array}{l}25(25.8) \\
50(51.5) \\
19(19.6)\end{array}$ \\
\hline Total PSA (ng/mL) & $\begin{array}{c}\quad<4 \\
4 \leq x<10 \\
\geq 10\end{array}$ & $\begin{array}{c}8(8.2) \\
60(61.9) \\
29(29.9)\end{array}$ \\
\hline
\end{tabular}


Table 1. Cont.

\begin{tabular}{ccc}
\hline Patient's Characteristics & Mean \pm SD & $N$ \\
\hline ISUP-GG & & \\
\hline \multirow{2}{*}{ Low Risk } & Group I & $32(33.0)$ \\
& Group II & $25(25.8)$ \\
\hline \multirow{2}{*}{ High Risk } & Group III & $23(23.7)$ \\
& Group IV & $10(10.3)$ \\
T pathological stage & Group V & $7(7.2)$ \\
& & \\
& $\leq$ T2a & $68(70.1)$ \\
N pathological stage & T3,T4 & $29(29.9)$ \\
& & \\
& NX & $57(58.8)$ \\
& N0 & $34(35.1)$ \\
& N1 & $6(6.2)$ \\
\hline
\end{tabular}

Abbreviations: BMI, body mass index; ISUP-GG, International Society of Urological Pathology Gleason Grade groups based on the Gleason score as follows: (Gleason score $\leq 6$-group I; $3+4$ $=7$ group II; $4+3=7$ group III; $4+4=8$-group IV; and 9-10-group V); PSA, prostate-specific antigen; $\mathrm{T}$ stage, Tumor category; $\mathrm{N}$ node, category. The bolded words differentiate the clinical and pathological characteristics from the rest of the table.
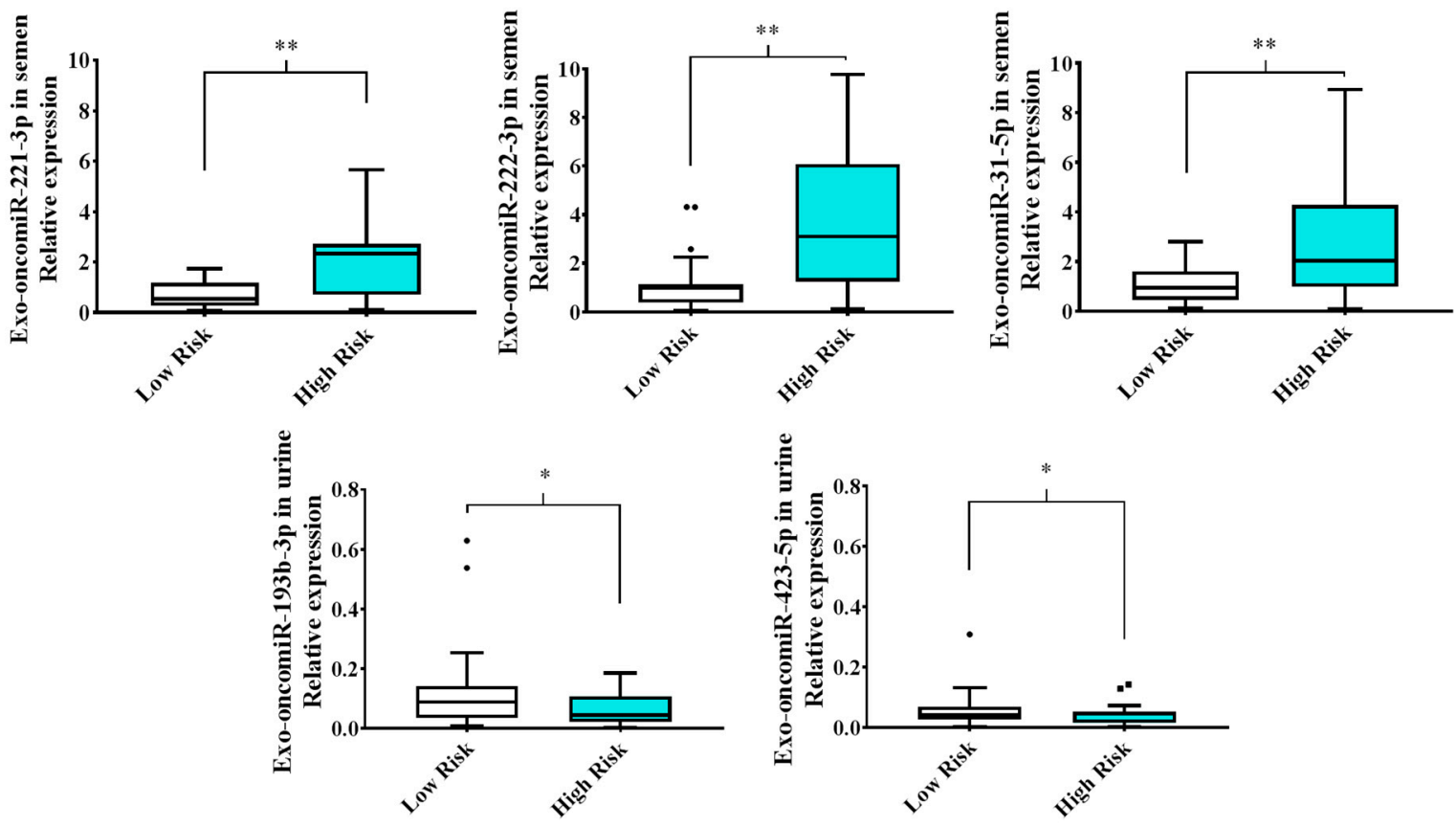

Figure 2. Exo-oncomiRNAs are differentially expressed in liquid biopsy from patients with prostate cancer. Box plots of relative expression of the 5 discriminatory exo-oncomiRNAs analyzed in semen and post-digital rectal examination urine liquid biopsies from patients with low- and high-risk PCa. Results are expressed as mean values \pm SD. Statistical differences between groups are indicated: ${ }^{*} p<0.05 ;{ }^{* *} p<0.01$.

We also examined for clinical and metabolic differences between the high-risk and low-risk groups (Table 2). Univariate analysis showed that only total PSA was significantly higher in the high-risk group than in the low-risk group $(p=0.007)$, whereas sTWEAK semen levels were significantly lower in the high-risk group than in the low-risk group $(p=0.009)$ (Table 2), as has been reported [11]. We then tested for correlations between the five differentially expressed exo-oncomiRNAs and clinical and metabolic parameters using Spearman's bivariate correlation coefficient test. The most relevant associations 
observed were the significant negative correlations between semen sTWEAK levels and the expression levels of exo-oncomiR-221-3p, exo-oncomiR-222-3p, and exo-oncomiR31-5p ( $\mathrm{r}=$ $-0.375 p=0.017, \mathrm{r}=-0.387 p=0.013$ and $\mathrm{r}=-0.364 p=0.021$, respectively) (Figure S2).

Table 2. Anthropometric and analytical characteristics according to ISUP-GG criteria.

\begin{tabular}{|c|c|c|c|}
\hline \multirow{5}{*}{ Patient's Stratification } & \multicolumn{2}{|c|}{ ISUP GG Classification } & \\
\hline & Low-Risk & High-Risk & \\
\hline & (Group I and II) & (Group III, IV and V) & \\
\hline & $N=57$ & $N=40$ & \\
\hline & Mean \pm SD & Mean \pm SD & $p$-Value \\
\hline \multicolumn{4}{|l|}{ Anthropometric parameters } \\
\hline Age (years) & $62.46 \pm 6.74$ & $64.96 \pm 5.52$ & 0.066 \\
\hline $\mathrm{BMI}\left(\mathrm{kg} / \mathrm{m}^{2}\right)$ & $27.97 \pm 4.07$ & $27.64 \pm 3.46$ & 0.718 \\
\hline Prostatic volume (c.c) & $48.68 \pm 24.56$ & $45.81 \pm 21$ & 0.687 \\
\hline \multicolumn{4}{|l|}{ Glycemic profile } \\
\hline Glucose (mmol/L) & $5.82 \pm 1.1$ & $6.29 \pm 2.26$ & 0.388 \\
\hline Insulin (pmol/L) & $89.36 \pm 58.39$ & $87.42 \pm 47.09$ & 0.841 \\
\hline HOMA-IR & $3.46 \pm 2.58$ & $3.67 \pm 2.71$ & 0.841 \\
\hline $\mathrm{HbA1c}(\%)$ & $5.74 \pm 0.64$ & $5.92 \pm 0.84$ & 0.364 \\
\hline \multicolumn{4}{|l|}{ Lipid profile } \\
\hline Cholesterol (mmol/L) & $5.03 \pm 1.06$ & $5.03 \pm 1.1$ & 0.957 \\
\hline HDL cholesterol (mmol/L) & $1.49 \pm 0.73$ & $1.42 \pm 0.39$ & 0.672 \\
\hline LDL cholesterol (mmol/L) & $3.28 \pm 1.3$ & $3 \pm 0.88$ & 0.503 \\
\hline Triglycerides (mmol/L) & $1.36 \pm 0.74$ & $1.55 \pm 0.96$ & 0.711 \\
\hline \multicolumn{4}{|l|}{ Hepatic profile } \\
\hline AST ( $\mu$ kat $/ \mathrm{L})$ & $0.39 \pm 0.19$ & $0.33 \pm 0.07$ & 0.171 \\
\hline ALT $(\mu \mathrm{kat} / \mathrm{L})$ & $0.42 \pm 0.22$ & $0.36 \pm 0.11$ & 0.402 \\
\hline GGT ( $\mu$ kat/L) & $0.7 \pm 0.85$ & $0.65 \pm 0.48$ & 0.887 \\
\hline \multicolumn{4}{|l|}{ Renal profile } \\
\hline Uric acid $(\mu \mathrm{mol} / \mathrm{L})$ & $368.05 \pm 83.1$ & $456.2 \pm 529.55$ & 0.376 \\
\hline Urea $(\mathrm{mmol} / \mathrm{L})$ & $14.26 \pm 3.23$ & $14.8 \pm 5.12$ & 0.808 \\
\hline Creatinine $(\mu \mathrm{mol} / \mathrm{L})$ & $85.83 \pm 18.44$ & $80.05 \pm 13.97$ & 0.072 \\
\hline \multicolumn{4}{|l|}{ Hormonal profile } \\
\hline SHBG (nmol/L) & $46.13 \pm 52.46$ & $40.02 \pm 16.36$ & 0.814 \\
\hline Testosterone (nmol/L) & $14.93 \pm 4.62$ & $13.55 \pm 5.63$ & 0.101 \\
\hline \multicolumn{4}{|l|}{ Tumoral marker } \\
\hline Total PSA $(\mu \mathrm{g} / \mathrm{L})$ & $7.71 \pm 4.8$ & $12.24 \pm 10.43$ & 0.007 \\
\hline \multicolumn{4}{|l|}{ Biofluid Biomarker profile } \\
\hline \multicolumn{4}{|c|}{ Semen cytokines (pg/mg of total protein) } \\
\hline STWEAK & $989.62 \pm 685.75$ & $617.25 \pm 447.57$ & 0.009 \\
\hline \multicolumn{4}{|c|}{ Exo-oncomiRNAs in semen-Relative expression levels } \\
\hline miR-221-3p & $0.75 \pm 0.6$ & $2.17 \pm 1.7$ & 0.002 \\
\hline $\mathrm{miR}-222-3 p$ & $2.01 \pm 2.79$ & $3.79 \pm 2.92$ & 0.006 \\
\hline miR-31-5p & $1.05 \pm 0.73$ & $2.75 \pm 2.27$ & 0.004 \\
\hline \multicolumn{4}{|c|}{ Exo-oncomiRNAs in urine-Relative expression levels } \\
\hline miR-193-3p & $0.12 \pm 0.12$ & $0.06 \pm 0.05$ & 0.037 \\
\hline $\operatorname{miR}-423-5 p$ & $0.05 \pm 0.05$ & $0.04 \pm 0.03$ & 0.034 \\
\hline
\end{tabular}

BMI, body mass index; HOMA-IR, homeostatic model assessment of insulin resistance; $\mathrm{HbA1c}$, Hemoglobin A1c; HDL, high-density lipoprotein; LDL, low-density lipoprotein; AST, aspartate aminotransferase; ALT, alanine aminotransferase; GGT, $\gamma$-Glutamyltransferase; SHBG, sex hormonebinding globulin; PSA, prostate-specific antigen; sTWEAK, soluble tumor necrosis factor-like weak inducer of apoptosis. 


\subsection{Semen Levels of Exo-oncomiR-221-3p May Help Identify an Aggressive Prostate Cancer Phenotype}

We developed a partial least square-discriminant analysis (PLS-DA) model to evaluate the potential of the five selected exo-oncomiRNAs plus PSA in serum, sTWEAK in semen, age, prostatic volume, and testosterone, for the stratification of patients. Cross-validation analyses showed that a one-component model had an accuracy of $72.02 \%\left(R^{2}=0.2073\right.$ and $\mathrm{Q}^{2}=0.1493$ ) (Figure S3a) indicating that is a good predictive model [32]. With regards to the importance of individual components, variable importance in projection (VIP) scores highlighted age, exo-oncomiR-222-3p in semen, exo-oncomiR-31-5p in semen, PSA in serum, sTWEAK in semen and exo-oncomiR-221-3p in semen as the most important variables (Figure 3). The VIP model estimated that exo-oncomiR-221-3p in semen and sTWEAK in semen had more influence than total PSA (Figure 3).

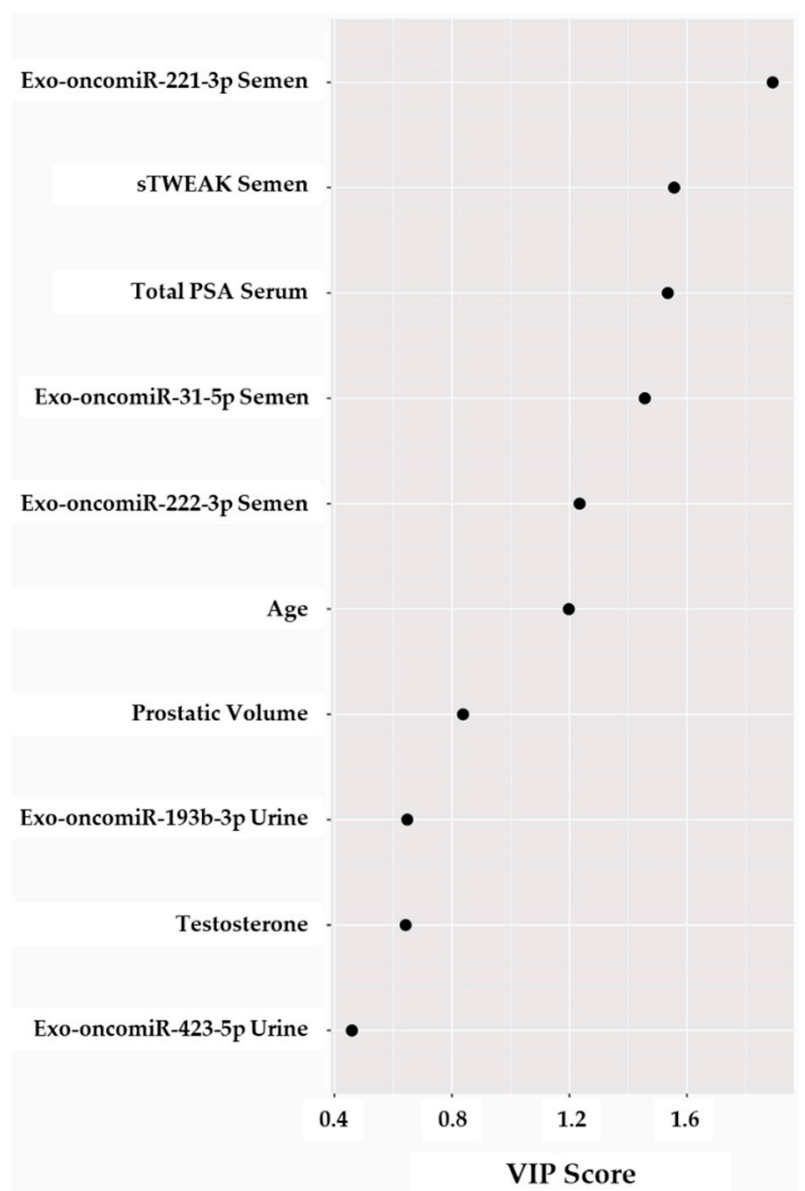

Figure 3. Variable importance in projection (VIP) scores. Selected variables: total PSA, testosterone, prostatic volume, age, sTWEAK in semen, exo-oncomiR-221-3p, exo-oncomiR222-3p, exo-oncomiR31$5 p$, exo-oncomiR-193-3p and exo-oncomiR-423-5p are shown in the model. Variables with scores close to or greater than 1 were considered important in the model.

Variables with VIP score $\geq 1$ were considered important in the model for determining PCa aggressiveness. To evaluate the usefulness of exo-oncomiRNAs as potential prognosis biomarkers of PCa aggressiveness in liquid biopsy, we performed logistic regression and receiver operating characteristic (ROC) curve analysis combining the following variables: exo-oncomiR-221-3p, exo-oncomiR-222-3p, exo-oncomiR-31-5p, total PSA, sTWEAK levels and age; Table 3 lists the different combinations. Results showed that the area under the curve (AUC) of each individual variable was below 0.8 . Thus, we used a multivariate regression model combining each potential biomarker to test which combination was more suitable for correct diagnosis. Notably, we observed that the presence of exo-oncomiR-221- 
$3 p$ outperformed the other individual variables alone or in combination. The best panel in our study to distinguish PCa aggressiveness was that composed by exo-oncomiR-221-3p, exo-oncomiR-222-3p and semen sTWEAK, which could correctly classify $87.5 \%$ of patients, with an AUC of 0.857 and with $85.7 \%$ specificity and $76.9 \%$ sensitivity (Table 3) (Figure S3).

Table 3. Exo-oncomiRNAs-based models as diagnostic classifiers.

\begin{tabular}{|c|c|c|c|c|c|c|c|c|}
\hline \multirow[b]{2}{*}{ ROC Model } & \multirow[b]{2}{*}{ AUC } & \multirow[b]{2}{*}{ Error } & \multicolumn{3}{|c|}{$95 \% \mathrm{CI}$} & \multirow[b]{2}{*}{$\begin{array}{l}\text { Sensivity } \\
(\%)\end{array}$} & \multirow[b]{2}{*}{$\begin{array}{l}\text { Specificity } \\
(\%)\end{array}$} & \multirow[b]{2}{*}{$\begin{array}{l}\text { \% Correct } \\
\text { Diagnosis }\end{array}$} \\
\hline & & & $p$-Value & Lower & Upper & & & \\
\hline Age & 0.610 & 0.058 & 0.066 & 0.496 & 0.724 & 85 & 75.4 & 62.9 \\
\hline Total PSA & 0.662 & 0.056 & 0.007 & 0.552 & 0.772 & 85 & 31.6 & 63.9 \\
\hline sTWEAK & 0.708 & 0.072 & 0.009 & 0.567 & 0.848 & 85.7 & 52.8 & 71.9 \\
\hline exo-oncomiR-221-3p & 0.79 & 0.078 & 0.002 & 0.638 & 0.943 & 86.7 & 55.6 & 78.6 \\
\hline exo-oncomiR-222-3p & 0.758 & 0.08 & 0.006 & 0.601 & 0.915 & 86.7 & 74.1 & 66.7 \\
\hline exo-oncomiR-31-5p & 0.768 & 0.082 & 0.004 & 0.607 & 0.929 & 86.7 & 48.1 & 76.2 \\
\hline Total PSA + Age & 0.704 & 0.054 & 0.001 & 0.597 & 0.810 & 85 & 70.2 & 67 \\
\hline Total PSA + sTWEAK & 0.738 & 0.072 & 0.003 & 0.597 & 0.879 & 85.7 & 47.2 & 71.9 \\
\hline Total PSA + exo-oncomiR-221-3p & 0.864 & 0.063 & $<0.001$ & 0.74 & 0.998 & 86.7 & 55.6 & 83.3 \\
\hline Total PSA + exo-oncomiR-222-3p & 0.78 & 0.071 & 0.003 & 0.641 & 0.919 & 86.7 & 55.6 & 73.8 \\
\hline Total PSA + exo-oncomiR-31-5p & 0.832 & 0.07 & $<0.001$ & 0.695 & 0.969 & 86.7 & 51.9 & 81 \\
\hline sTWEAK + Age & 0.709 & 0.069 & 0.009 & 0.574 & 0.844 & 85.7 & 50 & 66.7 \\
\hline sTWEAK + exo-oncomiR-221-3p & 0.841 & 0.073 & $<0.001$ & 0.698 & 0.983 & 85.7 & 69.2 & 82.5 \\
\hline sTWEAK + exo-oncomiR-222-3p & 0.745 & 0.086 & 0.012 & 0.576 & 0.913 & 85.7 & 42.3 & 70 \\
\hline sTWEAK + exo-oncomiR-31-5p & 0.808 & 0.077 & 0.001 & 0.657 & 0.958 & 85.7 & 61.5 & 77.5 \\
\hline exo-oncomiR-221-3p + Age & 0.802 & 0.077 & 0.001 & 0.651 & 0.954 & 86.7 & 33.3 & 76.2 \\
\hline $\begin{array}{l}\text { exo-oncomiR-221-3p + } \\
\text { exo-oncomiR-222-3p }\end{array}$ & 0.802 & 0.078 & 0.001 & 0.65 & 0.955 & 86.7 & 63 & 76.2 \\
\hline $\begin{array}{l}\text { exo-oncomiR-221-3p + } \\
\text { exo-oncomiR-31-5p }\end{array}$ & 0.8 & 0.079 & 0.001 & 0.646 & 0.954 & 86.7 & 55.6 & 81 \\
\hline exo-oncomiR-222-3p + Age & 0.751 & 0.081 & 0.008 & 0.592 & 0.909 & 86.7 & 66.7 & 73.8 \\
\hline $\begin{array}{l}\text { exo-oncomiR-222-3p + } \\
\text { exo-oncomiR-31-5p }\end{array}$ & 0.8 & 0.077 & 0.001 & 0.649 & 0.951 & 86.7 & 55.6 & 81 \\
\hline exo-oncomiR-31-5p + Age & 0.778 & 0.078 & 0.003 & 0.625 & 0.930 & 86.7 & 44.4 & 73.8 \\
\hline Total PSA + sTWEAK + Age & 0.746 & 0.067 & 0.002 & 0.614 & 0.878 & 85.7 & 44.4 & 73.7 \\
\hline $\begin{array}{l}\text { Total PSA + sTWEAK + } \\
\text { exo-oncomiR-221-3p }\end{array}$ & 0.863 & 0.068 & $<0.001$ & 0.73 & 0.996 & 85.7 & 69.2 & 85 \\
\hline $\begin{array}{l}\text { Total PSA + sTWEAK + } \\
\text { exo-oncomiR-222-3p }\end{array}$ & 0.758 & 0.086 & 0.008 & 0.59 & 0.926 & 85.7 & 46.2 & 75 \\
\hline $\begin{array}{l}\text { Total PSA + sTWEAK + } \\
\text { exo-oncomiR-31-5p }\end{array}$ & 0.824 & 0.076 & 0.001 & 0.675 & 0.974 & 85.7 & 73.1 & 77.5 \\
\hline $\begin{array}{l}\text { Total PSA + exo-oncomiR-221-3p + } \\
\text { Age }\end{array}$ & 0.889 & 0.056 & $<0.001$ & 0.780 & 0.998 & 85.7 & 37 & 83.3 \\
\hline $\begin{array}{l}\text { Total PSA + exo-oncomiR-221-3p + } \\
\text { exo-oncomiR-222-3p }\end{array}$ & 0.872 & 0.06 & $<0.001$ & 0.755 & 0.988 & 86.7 & 59.3 & 83.3 \\
\hline $\begin{array}{l}\text { Total PSA + exo-oncomiR-221-3p + } \\
\text { exo-oncomiR-31-5p }\end{array}$ & 0.854 & 0.067 & $<0.001$ & 0.724 & 0.985 & 86.7 & 51.9 & 83.3 \\
\hline $\begin{array}{l}\text { Total PSA + exo-oncomiR-222-3p + } \\
\text { Age }\end{array}$ & 0.840 & 0.064 & $<0.001$ & 0.714 & 0.965 & 86.7 & 37 & 83.3 \\
\hline $\begin{array}{l}\text { Total PSA + exo-oncomiR-222-3p + } \\
\text { exo-oncomiR-31-5p }\end{array}$ & 0.849 & 0.069 & $<0.001$ & 0.713 & 0.985 & 86.7 & 59.3 & 83.3 \\
\hline $\begin{array}{l}\text { Total PSA + exo-oncomiR-31-5p + } \\
\text { Age }\end{array}$ & 0.862 & 0.061 & $<0.001$ & 0.743 & 0.981 & 86.7 & 37 & 83.3 \\
\hline $\begin{array}{l}\text { sTWEAK + exo-oncomiR-221-3p + } \\
\text { Age }\end{array}$ & 0.854 & 0.067 & $<0.001$ & 0.723 & 0.986 & 85.7 & 23.7 & 77.5 \\
\hline $\begin{array}{l}\text { sTWEAK + exo-oncomiR-221-3p + } \\
\text { exo-oncomiR-222-3p }\end{array}$ & 0.857 & 0.069 & $<0.001$ & 0.721 & 0.993 & 85.7 & 76.9 & 87.5 \\
\hline
\end{tabular}


Table 3. Cont.

\begin{tabular}{|c|c|c|c|c|c|c|c|c|}
\hline \multirow[b]{2}{*}{ ROC Model } & \multicolumn{7}{|c|}{$95 \% \mathrm{CI}$} & \multirow[b]{2}{*}{$\begin{array}{l}\text { \% Correct } \\
\text { Diagnosis }\end{array}$} \\
\hline & AUC & Error & $p$-Value & Lower & Upper & $\begin{array}{l}\text { Sensivity } \\
(\%)\end{array}$ & $\begin{array}{l}\text { Specificity } \\
(\%)\end{array}$ & \\
\hline $\begin{array}{l}\text { sTWEAK + exo-oncomiR-221-3p + } \\
\text { exo-oncomiR-31-5p }\end{array}$ & 0.841 & 0.073 & $<0.001$ & 0.698 & 0.983 & 85.7 & 69.2 & 82.5 \\
\hline $\begin{array}{l}\text { sTWEAK + exo-oncomiR-222-3p + } \\
\text { Age }\end{array}$ & 0.764 & 0.078 & 0.006 & 0.611 & 0.917 & 85.7 & 50 & 72.5 \\
\hline $\begin{array}{l}\text { sTWEAK + exo-oncomiR-222-3p + } \\
\text { exo-oncomiR-31-5p }\end{array}$ & 0.83 & 0.073 & 0.001 & 0.687 & 0.972 & 85.7 & 53.8 & 82.5 \\
\hline $\begin{array}{l}\text { sTWEAK + exo-oncomiR-31-5p + } \\
\text { Age }\end{array}$ & 0.821 & 0.074 & 0.001 & 0.677 & 0.966 & 85.7 & 34.6 & 75 \\
\hline $\begin{array}{l}\text { exo-oncomiR-221-3p + } \\
\text { exo-oncomiR-222-3p + } \\
\text { exo-oncomiR-31-5p }\end{array}$ & 0.807 & 0.076 & 0.001 & 0.658 & 0.956 & 86.7 & 51.9 & 83.3 \\
\hline $\begin{array}{l}\text { exo-oncomiR-221-3p + } \\
\text { exo-oncomiR-222-3p + Age }\end{array}$ & 0.820 & 0.074 & 0.001 & 0.675 & 0.965 & 86.7 & 25.9 & 76.2 \\
\hline $\begin{array}{l}\text { exo-oncomiR-221-3p + } \\
\text { exo-oncomiR-31-5p + Age }\end{array}$ & 0.812 & 0.074 & 0.001 & 0.668 & 0.957 & 86.7 & 37 & 78.6 \\
\hline $\begin{array}{l}\text { exo-oncomiR-222-3p + } \\
\text { exo-oncomiR-31-5p + Age }\end{array}$ & 0.802 & 0.075 & 0.001 & 0.655 & 0.950 & 86.7 & 44 & 78.6 \\
\hline $\begin{array}{l}\text { Total PSA + sTWEAK + } \\
\text { exo-oncomiR-221-3p + } \\
\text { exo-oncomiR-222-3p }\end{array}$ & 0.86 & 0.071 & $<0.001$ & 0.721 & 0.999 & 85.7 & 69.2 & 85 \\
\hline $\begin{array}{l}\text { Total PSA + sTWEAK + } \\
\text { exo-oncomiR-221-3p + } \\
\text { exo-oncomiR-31-5p }\end{array}$ & 0.86 & 0.069 & $<0.001$ & 0.724 & 0.995 & 85.7 & 69.2 & 85 \\
\hline $\begin{array}{l}\text { Total PSA + sTWEAK + } \\
\text { exo-oncomiR-222-3p + } \\
\text { exo-oncomiR-31-5p }\end{array}$ & 0.83 & 0.076 & 0.001 & 0.682 & 0.978 & 85.7 & 65.4 & 82.5 \\
\hline $\begin{array}{l}\text { Age + Total PSA + sTWEAK + } \\
\text { exo-oncomiR-221-3p }\end{array}$ & 0.879 & 0.62 & $<0.001$ & 0.757 & 1 & 85.7 & 23.1 & 85 \\
\hline $\begin{array}{l}\text { Age + Total PSA + sTWEAK + } \\
\text { exo-oncomiR-222-3p }\end{array}$ & 0.808 & 0.074 & 0.001 & 0.662 & 0.953 & 85.7 & 50 & 82.5 \\
\hline $\begin{array}{l}\text { Age + Total PSA + sTWEAK + } \\
\text { exo-oncomiR-31-5p }\end{array}$ & 0.849 & 0.069 & $<0.001$ & 0.715 & 0.983 & 85.7 & 53.2 & 82.5 \\
\hline $\begin{array}{l}\text { Age }+ \text { Total PSA }+ \\
\text { exo-oncomiR-221-3p }+ \\
\text { exo-oncomiR-222-3p }\end{array}$ & 0.894 & 0.053 & $<0.001$ & 0.789 & 0.998 & 86.7 & 56.7 & 83.3 \\
\hline $\begin{array}{l}\text { Age }+ \text { Total PSA }+ \\
\text { exo-oncomiR-221-3p + } \\
\text { exo-oncomiR-31-5p }\end{array}$ & 0.879 & 0.059 & $<0.001$ & 0.764 & 0.994 & 86.7 & 54.3 & 83.3 \\
\hline $\begin{array}{l}\text { Age + Total PSA + } \\
\text { exo-oncomiR-222-3p + } \\
\text { exo-oncomiR-31-5p }\end{array}$ & 0.867 & 0.059 & $<0.001$ & 0.752 & 0.982 & 86.7 & 49.2 & 81 \\
\hline $\begin{array}{l}\text { Age + sTWEAK + } \\
\text { exo-oncomiR-221-3p + } \\
\text { exo-oncomiR-222-3p }\end{array}$ & 0.868 & 0.061 & $<0.001$ & 0.748 & 0.988 & 86.7 & 46.5 & 80 \\
\hline $\begin{array}{l}\text { Age + sTWEAK + } \\
\text { exo-onxomiR-221-3p + } \\
\text { exo-oncomiR-31-5p }\end{array}$ & 0.857 & 0.067 & $<0.001$ & 0.726 & 0.988 & 85.7 & 76.9 & 80 \\
\hline $\begin{array}{l}\text { Age + sTWEAK + } \\
\text { exo-oncomiR-222-3p + } \\
\text { exo-oncomiR-31-5p }\end{array}$ & 0.832 & 0.070 & 0.001 & 0.695 & 0.969 & 86.7 & 46.5 & 80 \\
\hline $\begin{array}{l}\text { Age + exo-oncomiR-221-3p + } \\
\text { exo-oncomiR-222-3p + } \\
\text { exo-oncomiR-31-5p }\end{array}$ & 0.820 & 0.072 & 0.001 & 0.678 & 0.962 & 86.7 & 48.1 & 81 \\
\hline
\end{tabular}


Table 3. Cont.

\begin{tabular}{|c|c|c|c|c|c|c|c|c|}
\hline \multirow[b]{2}{*}{ ROC Model } & \multicolumn{7}{|c|}{$95 \% \mathrm{CI}$} & \multirow[b]{2}{*}{$\begin{array}{l}\% \text { Correct } \\
\text { Diagnosis }\end{array}$} \\
\hline & AUC & Error & $p$-Value & Lower & Upper & $\begin{array}{c}\text { Sensivity } \\
(\%)\end{array}$ & $\begin{array}{c}\text { Specificity } \\
(\%)\end{array}$ & \\
\hline $\begin{array}{l}\text { Total PSA + exo-oncomiR-221-3p + } \\
\text { exo-oncomiR-222-3p + } \\
\text { exo-oncomiR-31-5p }\end{array}$ & 0.874 & 0.061 & $<0.001$ & 0.754 & 0.995 & 86.7 & 55.6 & 83.3 \\
\hline $\begin{array}{l}\text { sTWEAK + exo-oncomiR-221-3p + } \\
\text { exo-oncomiR-222-3p + } \\
\text { exo-oncomiR-31-5p }\end{array}$ & 0.86 & 0.068 & $<0.001$ & 0.726 & 0.994 & 85.7 & 73.1 & 85 \\
\hline $\begin{array}{l}\text { Total PSA + sTWEAK + } \\
\text { exo-oncomiR-221-3p + } \\
\text { exo-oncomiR-222-3p + } \\
\text { exo-oncomiR-31-5p }\end{array}$ & 0.865 & 0.069 & $<0.001$ & 0.73 & 1 & 85.7 & 69.2 & 85 \\
\hline $\begin{array}{l}\text { Age }+ \text { Total PSA }+ \text { sTWEAK }+ \\
\text { exo-oncomiR-221-3p }+ \\
\text { exo-oncomiR-222-3p }\end{array}$ & 0.879 & 0.062 & $<0.001$ & 0.757 & 1 & 86.7 & 70.4 & 87.5 \\
\hline $\begin{array}{l}\text { Age }+ \text { Total PSA }+ \text { sTWEAK }+ \\
\text { exo-oncomiR-221-3p }+ \\
\text { exo-oncomiR-31-5p }\end{array}$ & 0.879 & 0.062 & $<0.001$ & 0.758 & 1 & 86.7 & 57.9 & 85 \\
\hline $\begin{array}{l}\text { Age }+ \text { Total PSA }+ \text { sTWEAK }+ \\
\text { exo-oncomiR-222-3p }+ \\
\text { exo-oncomiR-31-5p }\end{array}$ & 0.857 & 0.065 & $<0.001$ & 0.729 & 0.985 & 85.7 & 58.3 & 85 \\
\hline $\begin{array}{l}\text { Age }+ \text { Total PSA }+ \\
\text { exo-oncomiR-221-3p + } \\
\text { exo-oncomiR-222-3p + } \\
\text { exo-oncomiR-31-5p }\end{array}$ & 0.896 & 0.053 & $<0.001$ & 0.793 & 0.999 & 86.7 & 57.9 & 83.3 \\
\hline $\begin{array}{l}\text { Age }+ \text { sTWEAK }+ \\
\text { exo-oncomiR-221-3p + } \\
\text { exo-oncomiR-222-3p + } \\
\text { exo-oncomiR-31-5p }\end{array}$ & 0.874 & 0.061 & $<0.001$ & 0.755 & 0.992 & 85.7 & 63.8 & 82.5 \\
\hline $\begin{array}{l}\text { Age }+ \text { Total PSA }+ \text { sTWEAK }+ \\
\text { exo-oncomiR-221-3p }+ \\
\text { exo-oncomiR-222-3p }+ \\
\text { exo-oncomiR-31-5p }\end{array}$ & 0.879 & 0.062 & $<0.001$ & 0.757 & 1 & 85.7 & 68.9 & 87.5 \\
\hline
\end{tabular}

Receiver operating characteristic (ROC) curve values showing the predictive efficiency for distinguishing PCa aggressiveness. Percentage of correct diagnostic values was obtained by multivariate models (backward stepwise, conditional method). AUC, area under the curve; $95 \%$ CI (confidence interval). The bolded words represent the ROC Models.

\subsection{TWEAK Modulates Potential Predicted Targets for oncomiR-221-3p}

Several studies have shown that oncomiR-221 and oncomiR-222 are dysregulated in many cancers [33], including PCa [34,35], which is in line with our findings showing deregulated exo-oncomiR-221-3p and exo-oncomiR-222-3p in semen liquid biopsy of PCa. In vitro analysis showed that oncomiR-221-3p expression was found significantly up-regulated by sTWEAK only in PC-3 cells, both internally and in secreted EVs, and not in LNCaP cells, indicating that sTWEAK can potentially modulate oncomiR221-3p downstream targets (Figure 4a). 
(a)

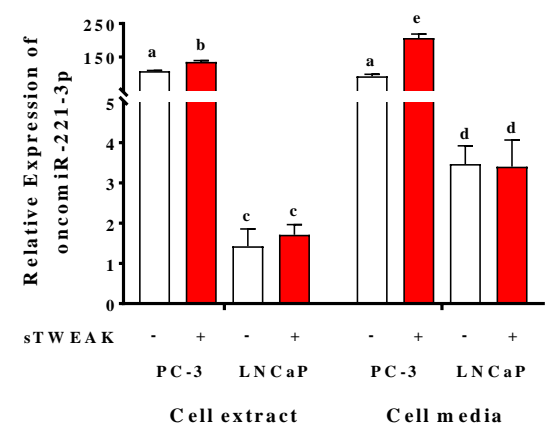

(b)

\begin{tabular}{c|cccc} 
& \multicolumn{4}{c}{$\begin{array}{c}\text { MicroRNA Target Prediction Databases } \\
\text { miRanda-miRSVR }\end{array}$} \\
Diana-MicroT-CDS & miRWalk \\
Targets Score & miRSVR & PhastCons & miTG & miRWalk \\
\hline TCF12 & -3.002 & $0.649 ; 0.816 ; 0.802 \#$ & 0.880 & 0.882 \\
SNAP23 & -1.192 & 0.643 & 0.770 & 0.846 \\
DPP8 & -1.187 & 0.650 & 0.827 & 0.962 \\
ARNT & -1.160 & 0.772 & 0.829 & 0.837 \\
NLK & -1.101 & 0.777 & 0.878 & 0.846 \\
ZFP36L2 & -0.587 & 0.578 & 0.961 & 0.923 \\
FNIP2 & -0.438 & 0.591 & 0.981 & 0.897 \\
ESR1 & -1.128 & $0.569 ; 0.719 \#$ & 0.768 & 0.955 \\
HIPK1 & -0.824 & 0.831 & 0.825 & 0.923 \\
SCARB2 & -0.426 & 0.692 & 0.798 & 0.846
\end{tabular}

(c)

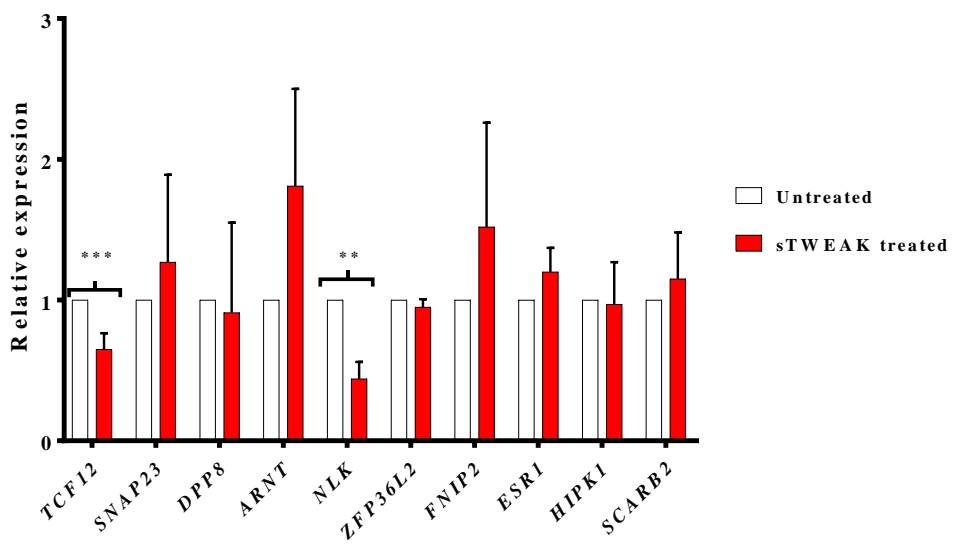

(d)

$\begin{array}{rrrrrrr}\text { STWEAK } & - & + & - & + & - & + \\ \text { iNC } & + & + & - & - & - & - \\ \text { i221-3p } & - & - & + & + & - & -\end{array}$
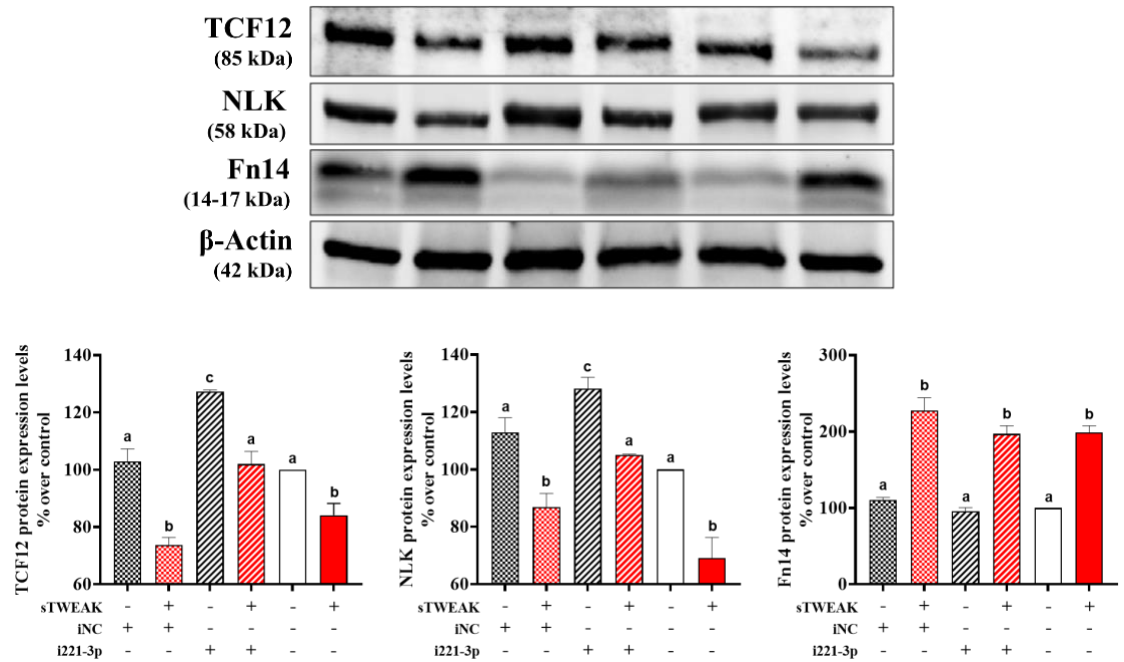

Figure 4. sTWEAK regulates oncomiR-221-3p expression and down-regulates NLK and TCF12 targets. (a) oncomiR-221-3p expression in PC-3 and LNCaP cell extracts and in extracellular vesicles (EVs) isolated from cell media. Different lettering over boxes indicates statistical differences. Significant differences are established at $p<0.05$. Data are expressed as mean \pm SEM $(n$ = 4 experiments). (b) Selected targets for oncomiR-221-3p by 3 different target prediction algorithms. ${ }^{\#}$ conserved elements in multiply-aligned sequences. (c) qRT-PCR mRNA expression of selected oncomiR-221-3p targets in PC-3 cells before and after treatment with sTWEAK. Significant differences: ${ }^{* *} p<0.01$; ${ }^{* * *} p<0.001$. Data are expressed as mean $\pm \operatorname{SEM}(n=6$ experiments). 
Transcription factor 12 (TCF12); synaptosome associated protein 23 (SNAP23); dipeptidyl peptidase 8 (DPP8); aryl hydrocarbon receptor nuclear translocator (ARNT); Nemo-like kinase (NLK); ZFP36 ring finger protein-like 2 (ZFP36L2); folliculin interacting protein 2 (FNIP2); estrogen receptor 1 (ESR1); homeodomain interacting protein kinase 1 (HIPK1); and scavenger receptor class B-ember 2 (SCARB2). (d) Expression of TCF12, NLK and Fn14 protein in PC-3 cells transfected with the oncomiR-221-3p inhibitor and further treated with sTWEAK. Representative Western blots are presented (top). The membranes were tested with the corresponding antibody. iNC: inhibitor negative control, i221-3p: inhibitor miR-221-3p; Nemo-like kinase (NLK); transcription factor 12 (TCF12); fibroblast growth factor 14 (Fn14). Relative protein expression levels are shown (bottom), which were normalized to the corresponding control $\beta$-actin. Different lettering over boxes indicates statistical differences. Significant differences are established at $p<0.05$. Data are expressed as mean \pm SEM ( $n=3$ experiments). Full-length blots and gels are presented in Figure S4.

To demonstrate a direct effect of sTWEAK on oncomiR-221-3p targets, we first searched for possible oncomiR-221-3p targets and selected only those shared by the miRanda, DianaMicroT-CDS and miRWalk databases. We obtained 69 genes with putative target sites for oncomiR-221-3p in their 3'untranslated regions. We then ranked the candidate genes by the miRSVR score (the lower the score, the stronger the match to the seed region); if two or more targets had a similar miRSVR score we considered the higher score from the Diana-microTCDS algorithm, miTG. With these criteria, we selected 10 possible targets implicated in cancer and/or inflammation (Figure 4b, Table S3), shared also by onco-miR222-3p, because both miRNAS are encoded in tandem and contain identical seed sequences separated by 727 bases [33] (Table S4). Of the 10 targets only NLK (Nemo-like kinase) and TCF12 (transcription factor 12) expression levels were found to be reduced in PC-3 cells after sTWEAK treatment for $24 \mathrm{~h}$ by real-time PCR (Figure $4 \mathrm{c}$ ) and Western blotting (Figure $4 \mathrm{~d}$ ). The stimulatory effect of sTWEAK was accompanied by the increased expression of its receptor Fn14 (Figure 4d).

Finally, we performed in vitro experiments using PC-3 cells and an oncomiR-221-3p inhibitor, which consistently influenced the expression of its target genes as demonstrated by the reduced expression of TCF12 and NLK proteins when compared with non-treated counterparts (Figure 4d). As anticipated, combined sTWEAK stimulation and oncomiR221-3p inhibition resulted in a significant down-regulation of NLK and TCF12 protein levels (Figure $4 \mathrm{~d}$ ). The detailed results of immunoblotting are shown in Figure S4.

\section{Discussion}

Histopathological biopsy analysis is a common method for the diagnosis of PCa. This procedure, however, only enables the analysis of part of the prostatic gland and, because of the typical multifocal nature of $\mathrm{PCa}$, information from a single biopsy is often insufficient and does not reflect the dynamics of the tumor in the prostate.

Diagnosis of cancer through the use of liquid biopsy has proven to be particularly useful as a non-invasive method of diagnosis and disease progression monitoring [36]. In a similar line, exosomal miRNAs isolated in the context of cancer, termed exo-oncomiRNAs, are promising biomarkers in part due to their stability in body fluids and ease of detection and quantification at low cost. Additionally, exo-oncomiRNAs have a very important role in modulating several critical cancer processes, including proliferation, migration, and angiogenesis, through their regulation of important target genes within the tumor environment [37].

Some exo-oncomiRNAs (e.g., miR-375, miR -21 and miR-141 [24,38]) from biofluids including blood and urine are known to have diagnostic and prognostic capacity in PCa. However, inconsistencies in identified, dysregulated exo-oncomiRNA profiles have been reported, likely due to a lack of standardized exosomal isolation and miRNA quantification techniques [23]. Despite these challenges, exo-oncomiRNAs remain highly promising biomarker candidates to aid in PCa diagnosis and prognosis. 
We previously established a non-invasive biomarker panel with high negative predictive value to classify PCa aggressiveness. Specifically, this biofluid signature comprised the following biomarkers: total PSA serum levels, semen levels of sTWEAK, fasting serum glycemia, and mRNA expression levels of $F n 14, K L K 2$ (a gene that encodes a protease that activates pre-PSA) and two chemokine receptors (CXCR2 and CCR3) in semen cell sediment. This panel can identify PCa aggressiveness with $90.9 \%$ success [11]. Although this panel could aid the clinical prognosis of PCa by outperforming the classical clinical biomarkers (age, T-classification, and total PSA serum levels), it requires the measurement of seven different biomarkers and uses two different biological samples-serum and semen.

In the aforementioned study, we observed that in patients with high-risk $\mathrm{PCa}$, the decrease in sTWEAK levels in semen was accompanied by an increase in Fn14 mRNA levels in seminal cell sediment, pointing to an active process of ligand-receptor interaction that may favor cell proliferation and migration, as described in PCa cell models $[17,18]$. Accordingly, the presence of TWEAK in PCa tumors could not only fuel tumor progression, but might also promote the secretion of exo-oncomiRNAs contained within EVs, which will likely have an impact on the tumor microenvironment.

In the search for an improved prognostic panel for PCa focusing on TWEAK-induced exo-oncomiRNAs, we show here that five exo-oncomiRNAs (exo-oncomiR-221-3p, exooncomiR-222-3p, exo-oncomiR-31-5p, exo-oncomiR-193b-3p, exo-oncomiR-423-5p) are significantly dysregulated between low- and high-risk PCa. VIP analysis of selected variables (including age, exo-oncomiRNA levels in semen and urine and, several analytical parameters) showed that variables with VIP scores greater than 1, considered of importance in the model for determining PCa aggressiveness, included only the three exo-oncomiRNAs expressed in semen (exo-oncomiR-221-3p, exo-oncomiR-222-3p and exo-oncomiR-31-5p). This finding may not be causal. Because $25 \%$ of semen is derived from prostatic tissue [39], its contents are more likely to contain prostate disease-specific exo-oncomiRNAs [29] than post-digital rectal examination urine samples [40].

After testing several logistic regression models followed by ROC analysis including the 3 selected biomarkers (exo-oncomiR-221-3p, exo-oncomiR-222-3p and sTWEAK), the measurements in semen liquid biopsy had the best prognostic accuracy (AUC $=0.857$, $p=0.001$ ) when compared with the ROC curve analysis using only serum PSA levels (AUC $=0.662, p<0.007$ ). This new model can outperform the classical PSA biomarker by $23.6 \%$ for a correct diagnosis, improving the classification efficacy up to $87.5 \%$. If we include the two selected exo-oncomiRNAs (exo-oncomiR-221-3p, exo-oncomiR-222-3p) plus PSA levels in serum, the model can predict PCa severity better than is commonly reported by PSA screening alone; however, the model composed of sTWEAK, exo-oncomiR-221-3p and exo-oncomiR-222-3p-all measured in semen-improves not only the percentage of positively diagnosed patients by $2.25 \%$, but increases the specificity by $8 \%$.

MiR-221 and miR-222 are encoded tandemly in chromosome Xp11.3, and are highly homologous miRNAs sharing the same "seed sequences" [33,41]. In vivo studies have demonstrated that miR-221/222 down-regulation impairs the growth of PCa xenografts, pointing to miR-221-3p as an oncogenic miRNA in PCa [42]. In the present study, we observed that the addition of exo-oncomiR-221-3p expression levels in semen improves all prognostic model panel combinations. miR-221 is overexpressed in a variety of epithelial cancers including breast, liver, bladder, pancreas, gastric, colorectal cancer, melanoma, papillary thyroid carcinoma and glioblastoma [33]. Additionally, miR-221 has been found to be related to cancer progression in cervical squamous cell carcinoma [43], confers adriamycin resistance in breast cancer [44], and is a biomarker in hepatocellular carcinoma [45], diffuse large B cell lymphomas [46] and lung adenocarcinoma [47].

Studies on the expression of miR-221 in PCa (which is referred to as mir-221-3p in MirBase), have used only PCa tissue [48,49] and have found the levels to be up-regulated. Here we show, for the first time to our knowledge that the expression levels of miR221-3p in PCa biofluids are higher in high-risk patients than in low-risk peers, and we additionally show that miR-221-3p is up-regulated in PC-3 secreted EVs and cell extracts. 
Mechanistically, in vitro studies have determined that miR-221-3p promotes proliferation of PCa cells [50].

MiR-221 directly targets NLK in neuroblastoma cells [51]. Accumulating evidence demonstrates that NLK has a pivotal role in cell proliferation, migration, invasion, and apoptosis by regulating a variety of transcriptional molecules [52]. NLK expression in PCa metastases is decreased in comparison with normal prostate epithelium and primary PCa [53]. Our findings show that oncomiR-221-3p inhibits NLK protein expression in PC-3 cells and that expression is further reduced by sTWEAK. An additional predicted and experimentally-demonstrated target for miR-221-3p is TCF12, a transcription factor member of the helix-loop-helix protein family found to be extensively expressed in many tissues [54]. As a target of miR-221, TCF12 has been related to survival after diagnosis of colon cancer [55], and there is evidence to suggest that TCF12 is involved in cell migration and differentiation [56]. Interestingly, the status of TCF12 has been found to be an independent predictor of biochemical recurrence-free survival in PCa [57]. We show here that miR-221-3p likely regulates TCF12 in PC-3 cells and its expression is, in turn, regulated by sTWEAK. While our findings point to the possibility that regulation of NLK or TCF12 might be a therapeutic approach against PCa tumors, further research and validation either in preclinical models or other established PCa cell lines will be needed to test their functional relevance in cell proliferation, invasion and chemosensitivity to cytotoxic agents.

Overall, our results reveal that TWEAK inflammation-induced exo-oncomiRNAs are components of an improved PCa prognostic panel based only on information obtained from a unique liquid biopsy, semen. Additionally, we reveal that a TWEAK inflammatory challenge in PCa cells can potentiate oncomiR-221-3p action.

\section{Materials and Methods}

\subsection{Cell Culture}

The PC-3 and LNCaP cell lines were purchased from Sigma-Aldrich (Barcelona, Spain). PC-3 cells were cultured in Ham's F-12K (Kaighn's) medium (1:1 mixture) with L-glutamate (Gibco, Fisher Scientific SL, Madrid, Spain), and LNCaP cells were cultured in RPMI 1640 medium supplemented with $1 \mathrm{mM}$ sodium pyruvate (Gibco). Cultures were also supplemented with $10 \%$ fetal bovine serum, $1 \times$ antibiotic-antimycotic solution (Gibco), and $5 \mu \mathrm{g} / \mathrm{mL}$ plasmocin, and cultured in a humidified $5 \% \mathrm{CO}_{2}$ atmosphere at $37^{\circ} \mathrm{C}$. Cells were grown in exosome-deprived serum overnight before stimulation for $24 \mathrm{~h}$ with 100 ng/mL human recombinant (hr) TWEAK (PeproTech, BioNova Cientifica, Barcelona, Spain).

\subsection{Extracellular Vesicle Isolation from Cell Culture Media and Exo-oncomiRNA Expression Profile Using TaqMan Low-Density Arrays}

Exosomes and other extracellular vesicles from cell culture media (PC-3 and LNCaP) were isolated and exo-oncomiRNAs were extracted using the exoRNeasy Serum/Plasma Maxi Kit (Qiagen, BioNova Cientifica, Madrid, Spain). For exo-oncomiRNA screening, the miRCURY LNA Universal RT microRNA PCR, Polyadenylation and cDNA Synthesis Kit (Exiqon, BioNova Cientifica, s.l. Madrid, Spain) was used for reverse transcription. cDNA was diluted and assayed by qRT-PCR according to the protocol in a 7900HT Fast Real-Time PCR System (Applied Biosystems, Thermo Fisher Scientic, Waltham, MA, USA). Each exo-oncomiRNA was assayed using ExiLENT SYBR Green Master Mix on the Human panel I+II, V5, miRCURY LNA miRNA miRNome PCR Panel (Qiagen) that included 752 mature human cancer-related miRNAs. Fluorescence readings and expression records of the microRNAs during the qRT-PCR were performed with the SDS 2.3 program (Applied Biosystems, Foster City, CA, USA). From the quantitative analysis by qRT-PCR of all miRNAs analyzed, we only considered those miRNAs that showed expression levels with a $\mathrm{Ct}<33$. Then, using the GeneGlobe program (Qiagen) [58], $\mathrm{C}_{\mathrm{T}}$ values for each sample were normalized to the arithmetic mean of the following reference miRNAs, hsa-miR423-5p, SNORD38B, SNORD49A, hsa-miR-191-5p, hsa-miR-103a-3p and U6 small nuclear 
RNA. The fold change expression of each exo-oncomiRNA was calculated with the formula $2^{-\Delta \Delta C t}$ where each miRNA, regardless of the condition, was first normalized to the $\mathrm{C}_{\mathrm{T}}$ of an endogenous control and then we calculated the $\Delta \Delta \mathrm{Ct}=\Delta \mathrm{Ct}$ sample treated sTWEAK $-\Delta \mathrm{Ct}$ untreated controls. The exo-oncomiRNAs with $p \leq 0.05$ when comparing cell type and condition and with an increase $\geq 1$.8-fold were considered for further analysis.

\subsection{Extracellular Vesicle Analysis}

Extracellular vesicles from culture media, post-digital rectal examination urine and semen were obtained using exoRNeasy Serum/Plasma Maxi Kit just before miRNA isolation by the addition of $500 \mu \mathrm{L}$ of elution buffer XE. The isolated EVs were further concentrated using a 100,000 Da cut-off concentrator (Amicon Ultra-0.5 mL Centrifugal Filters, Millipore). Samples were then ultrasonicated 3 times during $1 \mathrm{~min}$ bouts. Total protein was quantified using the BCA method (Pierce). A total amount of $10 \mu \mathrm{g}$ EV protein and $10 \mu \mathrm{g}$ total PC-3 cell extract were loaded on 4-15\% SDS-PAGE gels and immunoblotted with polyclonal rabbit antibodies against: EXOAB-CD9A1, EXOAB-CD81A-1, EXOAB-CD63A-1, EXOABHsp70A1, EXOAB-TSG101-1 (System Biology, Palo Alto, CA, USA), and the mouse monoclonal antibody for tubulin (Thermo Fisher Scientific, Waltham, MA, USA). HRP-conjugated goat anti-mouse or anti-rabbit (both from SBI) were used as secondary antibodies. All Western blots were developed with SuperSignal West Femto chemiluminescen substrate (Pierce Biotechnology, Boston, MA, USA) and visualized with the VersaDoc imaging system and Quantity One software (Bio-Rad, Barcelona, Spain) (Supplementary Materials).

\subsection{Transmission Electron Microscopy Analysis}

EVs were placed on carbon-coated copper grids (200 mesh), allowed to dry, and incubated in osmium tetroxide vapors for 15-30 min. TEM images were collected using a JEOL 1011 transmission electron microscope operating at $80 \mathrm{kV}$ with a megaview III camera.

\subsection{Patients}

Our studied patient cohort comprised 97 consecutive patients with PCa who had undergone radical prostatectomy by open surgery at the University Hospital Joan XXIII, Tarragona, between 2015 and 2019-laparoscopic or robotic surgery (intraperitoneal or extraperitoneal-with or without bilateral ilio-obturator lymphadenectomy, according to the estimated risk of lymphadenopathy based on the Briganti nomogram [56]. Patients were stratified according to the 2014 ISUP-GG and TNM classification $[57,58]$. Patients were stratified into two categories: low-risk (ISUP Group I and II) and high-risk (ISUP Groups III, IV and V). Written informed consent prior to their inclusion was provided by all patients. The study was approved by our local ethics committee and performed according to the provisions of the Declaration of Helsinki (Biomedical Research Law 14/2007, Royal Decree of Biobanks 1716/2011, Organic Law15/1999 of September 13 Protection of Personal Data) [11]. Clinical parameters, tumor aggressiveness, and metabolic status of all patients were documented. All methods were approved and performed in accordance with guidelines and regulations of the Ethical Committee for Clinical Research (CEIm) from Pere Virgili Research Institute (Ref. CEim171/2017) (http:/ / www.iispv.cat/ plataformes_de_suport/en_comite-iispv.html). Patient's inclusion criteria were as follows: older than 18 years, diagnosed with PCa by prostate biopsy in our center or any other, and treated by radical prostatectomy in our center. Exclusion criteria were patients with a previous history of cancer, patients older than 75 years, and those who had received any prior treatment before radical prostatectomy for PCa, as described [11].

\subsection{Analytical Methods}

Plasma glucose, cholesterol, triglyceride, high-density lipoprotein cholesterol, insulin levels and hepatic profile and renal profile was performed as described [59]. Levels of sTWEAK in semen were determined in duplicate using commercially available human 
enzyme-linked immunosorbent assay (ELISA) DuoSet Kits (R\&D Systems Europe, Abingdon, UK).

\subsection{Sample Processing}

Serum/plasma: blood samples were collected after a fast of at least $12 \mathrm{~h}$, or $2 \mathrm{~h}$ after an oral glucose tolerance test. Samples were centrifuged at $4{ }^{\circ} \mathrm{C}$ and stored at $-80^{\circ} \mathrm{C}$.

Post-digital rectal examination urine: urine samples were collected prior to prostate biopsy or surgical intervention. Samples were centrifuged $\left(2000 \times g, 10 \mathrm{~min}, 4{ }^{\circ} \mathrm{C}\right)$, and stored at $-80^{\circ} \mathrm{C}$.

Semen: semen samples were centrifuged at $2000 \times g$ for $15 \mathrm{~min}$ at $22^{\circ} \mathrm{C}$ to separate spermatozoa from semen plasma, and the supernatant (semen plasma) was stored at $-80^{\circ} \mathrm{C}$.

All samples were processed and stored at the Institut d'Investigació Sanitària Pere Virgili (IISPV) BioBanc (B.0000853 + B.0000854) integrated in the Spanish National Biobanks Platform (PT13/0010/0029 and PT13/0010/0062) for its collaboration.

\subsection{Extracellular Vesicles Extraction from Liquid Biopsy and Exo-onocomiRNA Quantitative Real-Time PCR Profiling}

Extracellular vesicles and exo-miRNAs were isolated and extracted from urine and semen samples using the exoRNeasy Serum/Plasma Maxi Kit or Midi Kit (Qiagen) [12]. The miRCURY LNA Universal RT microRNA PCR, Polyadenylation and cDNA Synthesis Kit (Exiqon, BioNova Cientifica, s.l. Madrid, Spain) was used for reverse transcription. The expression profile of the 14 selected exo-oncomiRNAs was further analyzed in urine and semen samples in duplicate, using individual primers on a 7900HT Fast Real-Time PCR System (Applied Biosystems). Data were analyzed by SDS 2.3 and RQ Manager 1.2 (Applied Biosystems) using the $2^{-\Delta \Delta C t}$ method. All values of $\mathrm{Ct}>35$ were excluded for further analysis.

\subsection{Target Search by Bioinformatic Analysis}

The targets of the selected exo-oncomiRNAs were searched using three target prediction software packages: (1) The miRanda algorithm (www.microRNA.org) was used to find potential target sites for miRNAs in the genomic sequence. From the miRanda algorithm results, we used the mirSVR score and PhastCons score to decipher which targets were potentially predicted. The mirSVR score is an estimate of the miRNA effect on the mRNA expression level; the more negative the score, the greater the inhibitory effect. PhastCons scores measure the conservation of nucleotide positions across vertebrates of any possible interaction; the higher the PhastCons value, the more conservative across vertebrates and the more important is the complementarity of the miRNA and the target [60]. (2) The Diana-MicroT-CDS predicts targets through the microT-CDS algorithm giving a miTG score, which is a general score for the predicted interaction. The closer the score is to 1, the greater the prediction confidence [61]. (3) Finally, we used the miRWalk platform. The calculated score is generated by executing the TarPmiR algorithm for miRNA target site prediction. The closer the score is to 1 , the greater is the confidence prediction, in the same way as Diana-MicroT-CDS [62].

Candidate targets with an miR-SVR score equal or to less than -0.1; a PhastCons value equal or greater to 0.56 and, an miTG and miRWalk score equal or greater to 0.8 were considered as potential targets of exo-oncomiR-221-3p.

\subsection{Functional Studies}

Functional studies were performed in PC-3 cells cultured in 6-well plates and grown at 90\% confluence. Cells were transfected using Lipofectamine 3000 (Invitrogen, Thermo Fisher Scientific, Waltham, MA, USA) and OPTI-MEM medium (Gibco) with a locked nucleic acid probe containing a specific sequence antisense oligonucleotide targeting exooncomiR-221-3p, miRCURY LNA exo-oncomiR-221-3p Power Inhibitor (Qiagen). A scrambled miRNA sequence, miRCURY LNA Power Inhibitor Control A (Qiagen), served as 
a negative control. Each condition was incubated for $24 \mathrm{~h}$ in a humidified $5 \% \mathrm{CO}_{2}$ atmosphere at $37^{\circ} \mathrm{C}$ before stimulation for $24 \mathrm{~h}$ with $100 \mathrm{ng} / \mathrm{mL}$ TWEAK (PeproTech) in serum-free media. After stimulation, cells were harvested for protein and RNA analysis. Expression analyses of target genes were performed using commercial individual primers on a 7900HT Fast Real-Time PCR System (Applied Biosystems). Protein analysis and Western blotting were performed using standard protocols. Nitrocellulose membranes were probed with the following primary antibodies that were purchased from Cell Signaling Technology (Danvers, MA, USA): TCF12/HEB (\#11825) and NLK (\#94350) and NF-kB2 p100 (\#4882). An anti- $\beta$-actin (A11126) antibody was purchased from Sigma-Aldrich. The standard molecular weight marker used was purchased from New England Biolabs Inc. (Herts, UK). Western blots were developed with SuperSignal West Femto chemiluminescen substrate (Pierce Biotechnology, Boston, MA, USA) and visualized with VersaDoc imaging system and Quantity One software (Bio-Rad) (Supplementary Materials).

\subsection{Statistical Analysis}

For in vitro assays, experimental results are presented as mean \pm standard error of the mean (SEM) of 3-4 experiments. Statistical significance was assessed with Student's $t$-test. Results with $p<0.05$ were considered statistically significant.

For human samples studies, the sample size was calculated to determine differences between exo-oncomiRNA expression levels in liquid biopsy with respect to the degree of aggressiveness of the tumor (low-risk/high-risk) in those patients diagnosed with PCa. We assumed a two-fold change difference between groups and identical standard deviation (SD) between the groups; therefore, a minimum of 35 patients was needed in each group (bilateral alpha error 0.05, power $90 \%$ ). Statistical analysis were performed as described [11]. Briefly, for anthropometric and clinical variables, data are expressed as mean \pm SD. Before statistical analysis, normal distribution was evaluated using Levene's test. The non-parametric Mann-Whitney U-test was used to analyze the differences in anthropometric and clinical data and absolute expression levels of the exomiR candidates between patients according to ISUP-GG-low-risk (Group I and II) and high-risk (Group III, Group IV, and Group V). A p-value less than 0.05 was considered statistically significant. Spearman's Rho test was used as a correlation analysis between anthropometric, clinical, and exo-miRNAs data. Partial least square discriminant analysis (PLS-DA) and VIP analysis models and binary logistic regression analysis were developed for selected variables. ROC curve analysis was performed to evaluate the best predictive model. The statistical software SPSS Statistics 24.0 (IBM, Madrid, Spain) package and R software (http:/ / cran.r-project.org) were used for analysis.

\section{Conclusions}

TWEAK, exo-oncomiR -221-3p and exo-oncomiR-222-3p are proposed as an improved PCa prognostic panel based on information obtained from a unique biofluid, semen. Further studies in larger cohorts of PCa will be needed as a next step to confirm/validate our panel before it can be adopted in clinical practice.

Supplementary Materials: The following are available online at https://www.mdpi.com/2072 -6694/13/2/250/s1. Table S1: 14 identified exo-oncomiRNAS, Table S2: Exo-oncomiRNAS nonsignificantly changed when comparing urine or semen biofluids from patients with PCa stratified by risk (low or high), Table S3: Role of elected exo-miR-221-3p targets in cancer, Table S4: List of the selected exo-oncomiR-221-3p and exo-oncomiR-222-3p target's scores, Figure S1: Complete Western blot (WB) results referring to Figure 1b, Figure S2: Spearman's correlation, Figure S3a,b: PLS-DA analysis, Figure S4: Complete WB results referring to Figure 4d.

Author Contributions: Conceptualization, M.R.C., X.R.-P., J.S.-T.; methodology, A.A.-C.; sample collection, review of clinical data and patient recruitment, M.A.-S., J.F.G.-F., J.V.-B. and S.M.-G.; writing-review and editing, M.R.C., X.R.-P., J.S.-T. and A.A.-C.; funding acquisition, M.R.C. and X.R.-P. All authors have read and agreed to the published version of the manuscript. 
Funding: This study was founded by Instituto de Salud Carlos III through projects PI17/00877, PI20/00418 (co-founded by the European Regional Development Fund/European Social Found; “A way to make future" / Investing in your future") and a grant awarded by Fundació Vallformosa "IV Premi Martí Via". No payment has been received to write this article by a pharmaceutical company or other agency.

Institutional Review Board Statement: The study was conducted according to the guidelines of the Declaration of Helsinki, and approved by the Institutional Review Board (or Ethics Committee) of Institut d'Investigació Sanitària Pere Virgili (Ref.CEim 171/2017).

Informed Consent Statement: Informed consent was obtained from all subjects involved in the study.

Data Availability Statement: The data presented in this study is contained within the article and the supplementary material.

Acknowledgments: The authors wish to particularly acknowledge the patients enrolled in this study for their participation and to the IISPV BioBanc (B.0000853 + B.0000854) integrated in the Spanish National Biobanks Platform (PT13/0010/0029 and PT13/0010/0062) for its collaboration.

Conflicts of Interest: The authors declare no conflict of interest.

\section{References}

1. Bray, F.; Ferlay, J.; Soerjomataram, I.; Siegel, R.L.; Torre, L.A.; Jemal, A. Global cancer statistics 2018: GLOBOCAN estimates of incidence and mortality worldwide for 36 cancers in 185 countries. CA. Cancer J. Clin. 2018, 68, 394-424. [CrossRef]

2. Markozannes, G.; Tzoulaki, I.; Karli, D.; Evangelou, E.; Ntzani, E.; Gunter, M.J.; Norat, T.; Ioannidis, J.P.; Tsilidis, K.K. Diet, body size, physical activity and risk of prostate cancer: An umbrella review of the evidence. Eur. J. Cancer 2016, 69, 61-69. [CrossRef] [PubMed]

3. Epstein, J.I.; Egevad, L.; Amin, M.B.; Delahunt, B.; Srigley, J.R.; Humphrey, P.A. The 2014 International Society of Urological Pathology (ISUP) Consensus Conference on Gleason Grading of Prostatic Carcinoma. Am. J. Surg. Pathol. 2015, 40, 1. [CrossRef] [PubMed]

4. Saini, S. PSA and beyond: Alternative prostate cancer biomarkers. Cell. Oncol. 2016, 39, 97-106. [CrossRef]

5. Di Meo, A.; Bartlett, J.; Cheng, Y.; Pasic, M.D.; Yousef, G.M. Liquid biopsy: A step forward towards precision medicine in urologic malignancies. Mol. Cancer 2017, 16, 1-14. [CrossRef]

6. Greten, F.R.; Grivennikov, S.I. Inflammation and Cancer: Triggers, Mechanisms, and Consequences. Immunity 2019, 51, 27-41. [CrossRef]

7. Winkles, J.A. The TWEAK-Fn14 cytokine-receptor axis: Discovery, biology and therapeutic targeting. Nat. Rev. Drug Discov. 2008, 7, 411-425. [CrossRef]

8. Maecker, H.; Varfolomeev, E.; Kischkel, F.; Lawrence, D.; LeBlanc, H.; Lee, W.; Hurst, S.; Danilenko, D.; Li, J.; Filvaroff, E.; et al. TWEAK attenuates the transition from innate to adaptive immunity. Cell 2005, 123, 931-944. [CrossRef]

9. Terra, X.; Gómez, D. External validation of sTWEAK as a prognostic noninvasive biomarker for head and neck squamous cell carcinoma. Head Neck 2014, 36, 1391. [CrossRef]

10. Avilés-Jurado, F.X.; Terra, X.; Gómez, D.; Flores, J.C.; Raventós, A.; Maymó-Masip, E.; León, X.; Serrano-Gonzalvo, V.; Vendrell, J.; Figuerola, E.; et al. Low blood levels of sTWEAK are related to locoregional failure in head and neck cancer. Eur. Arch. Oto-Rhino-Laryngol. 2015, 272, 1733-1741. [CrossRef]

11. Ruiz-Plazas, X.; Rodríguez-Gallego, E.; Alves, M.; Altuna-Coy, A.; Lozano-Bartolomé, J.; Portero-Otin, M.; García-Fontgivell, J.F.; Martínez-González, S.; Segarra, J.; Chacón, M.R. Biofluid quantification of TWEAK/Fn14 axis in combination with a selected biomarker panel improves assessment of prostate cancer aggressiveness. J. Transl. Med. 2019, 17, 1-13. [CrossRef] [PubMed]

12. Enderle, D.; Spiel, A.; Coticchia, C.M.; Berghoff, E.; Mueller, R.; Schlumpberger, M.; Sprenger-Haussels, M.; Shaffer, J.M.; Lader, E.; Skog, J.; et al. Characterization of RNA from exosomes and other extracellular vesicles isolated by a novel spin column-based method. Wiley Interdiscip. Rev. RNA 2012, 3, 286-293. [CrossRef] [PubMed]

13. Ruivo, C.F.; Adem, B.; Silva, M.; Melo, S.A. The biology of cancer exosomes: Insights and new perspectives. Cancer Res. 2017, 77, 6480-6488. [CrossRef] [PubMed]

14. Tkach, M.; Théry, C. Communication by Extracellular Vesicles: Where We Are and Where We Need to Go. Cell 2016, 164, 1226-1232. [CrossRef] [PubMed]

15. Pan, J.; Ding, M.; Xu, K.; Yang, C.; Mao, L.J. Exosomes in diagnosis and therapy of prostate cancer. Oncotarget 2017, 8, 97693-97700. [CrossRef]

16. Huang, C.; Luo, W.-F.; Ye, Y.-F.; Lin, L.; Wang, Z.; Luo, M.-H.; Song, Q.-D.; He, X.-P.; Chen, H.-W.; Kong, Y.; et al. Characterization of Inflammatory Factor-Induced Changes in Mesenchymal Stem Cell Exosomes and Sequencing Analysis of Exosomal microRNAs. World J. Stem Cells 2019, 11, 859-890. [CrossRef] [PubMed] 
17. Huang, M.; Narita, S.; Tsuchiya, N.; Ma, Z.; Numakura, K.; Obara, T.; Tsuruta, H.; Saito, M.; Inoue, T.; Horikawa, Y.; et al. Overexpression of Fn14 promotes androgen-independent prostate cancer progression through MMP-9 and correlates with poor treatment outcome. Carcinogenesis 2011, 32, 1589-1596. [CrossRef]

18. Yin, J.; Morrissey, C.; Barrett, B.; Corey, E.; Ylaya, K.; Hewitt, S.; Fang, L.; Tillman, H.; Lake, R.; Vessella, R.; et al. AR-Regulated TWEAK-FN14 Pathway Promotes Prostate Cancer Bone Metastasis. Cancer Res. 2014, 74, 4306-4317. [CrossRef]

19. Hu, Y.; Li, D.; Wu, A.; Qiu, X.; Di, W.; Huang, L.; Qiu, L. TWEAK-stimulated macrophages inhibit metastasis of epithelial ovarian cancer via exosomal shuttling of microRNA. Cancer Lett. 2017, 393, 60-67. [CrossRef]

20. Valadi, H.; Ekström, K.; Bossios, A.; Sjöstrand, M.; Lee, J.J.; Lötvall, J.O. Exosome-mediated transfer of mRNAs and microRNAs is a novel mechanism of genetic exchange between cells. Nat. Cell Biol. 2007, 9, 654-659. [CrossRef]

21. Svoronos, A.A.; Engelman, D.M.; Slack, F.J. OncomiR or tumor suppressor? The duplicity of MicroRNAs in cancer. Cancer Res. 2016, 76, 3666-3670. [CrossRef] [PubMed]

22. Tang, Z.; Li, D.; Hou, S.; Zhu, X. The cancer exosomes: Clinical implications, applications and challenges. Int. J. Cancer 2020, 146, 2946-2959. [CrossRef] [PubMed]

23. Wang, J.; Ni, J.; Beretov, J.; Thompson, J.; Graham, P.; Li, Y. Exosomal microRNAs as liquid biopsy biomarkers in prostate cancer. Crit. Rev. Oncol. Hematol. 2020, 145, 4-10. [CrossRef] [PubMed]

24. Huang, X.; Yuan, T.; Liang, M.; Du, M.; Xia, S.; Dittmar, R.; Wang, D.; See, W.; Costello, B.A.; Quevedo, F.; et al. Exosomal miR-1290 and miR-375 as Prognostic Markers in Castration-resistant Prostate Cancer. Eur. Urol. 2015, 67, 33-41. [CrossRef] [PubMed]

25. Endzelinš, E.; Berger, A.; Melne, V.; Bajo-Santos, C.; Sobolevska, K.; Abols, A.; Rodriguez, M.; Šantare, D.; Rudnickiha, A.; Lietuvietis, V.; et al. Detection of circulating miRNAs: Comparative analysis of extracellular vesicle-incorporated miRNAs and cell-free miRNAs in whole plasma of prostate cancer patients. BMC Cancer 2017, 17, 1-13. [CrossRef]

26. Foj, L.; Ferrer, F.; Serra, M.; Arévalo, A.; Gavagnach, M.; Giménez, N.; Filella, X. Exosomal and Non-Exosomal Urinary miRNAs in Prostate Cancer Detection and Prognosis. Prostate 2017, 77, 573-583. [CrossRef] [PubMed]

27. Li, Z.; Ma, Y.Y.; Wang, J.; Zeng, X.F.; Li, R.; Kang, W.; Hao, X.K. Exosomal microRNA-141 is upregulated in the serum of prostate cancer patients. OncoTargets Ther. 2015, 9, 139-148. [CrossRef]

28. Wani, S.; Kaul, D.; Mavuduru, R.S.; Kakkar, N.; Bhatia, A. Urinary-exosomal miR-2909: A novel pathognomonic trait of prostate cancer severity. J. Biotechnol. 2017, 259, 135-139. [CrossRef]

29. Barceló, M.; Castells, M.; Bassas, L.; Vigués, F.; Larriba, S. Semen miRNAs Contained in Exosomes as Non-Invasive Biomarkers for Prostate Cancer Diagnosis. Sci. Rep. 2019, 9, 1-16. [CrossRef]

30. Lee, J.M.; Im, G.-I. PTHrP isoforms have differing effect on chondrogenic differentiation and hypertrophy of mesenchymal stem cells. Biochem. Biophys. Res. Commun. 2012, 421, 819-824. [CrossRef]

31. Konoshenko, M.Y.; Lekchnov, E.A.; Vlassov, A.V.; Laktionov, P.P. Isolation of Extracellular Vesicles: General Methodologies and Latest Trends. Biomed. Res. Int. 2018, 2018, 8545347. [CrossRef] [PubMed]

32. Fay, M.P.; Shih, J.H. Permutation tests using estimated distribution functions. J. Am. Stat. Assoc. 1998, 93, 387-396. [CrossRef]

33. Song, J.; Ouyang, Y.; Che, J.; Li, X.; Zhao, Y.; Yang, K.; Zhao, X.; Chen, Y.; Fan, C.; Yuan, W. Potential value of miR-221/222 as diagnostic, prognostic, and therapeutic biomarkers for diseases. Front. Immunol. 2017, 8, 1-9. [CrossRef]

34. Goto, Y.; Kojima, S.; Nishikawa, R.; Kurozumi, A.; Kato, M.; Enokida, H.; Matsushita, R.; Yamazaki, K.; Ishida, Y.; Nakagawa, M.; et al. MicroRNA expression signature of castration-resistant prostate cancer: The microRNA-221/222 cluster functions as a tumour suppressor and disease progression marker. Br. J. Cancer 2015, 113, 1055-1065. [CrossRef]

35. Coarfa, C.; Fiskus, W.; Eedunuri, V.K.; Rajapakshe, K.; Foley, C.; Chew, S.A.; Shah, S.S.; Geng, C.; Shou, J.; Mohamed, J.S.; et al. Comprehensive proteomic profiling identifies the androgen receptor axis and other signaling pathways as targets of microRNAs suppressed in metastatic prostate cancer. Oncogene 2016, 35, 2345-2356. [CrossRef]

36. Lobo, J.; Gillis, A.J.; van den Berg, A.; Dorssers, L.C.; Belge, G.; Dieckmann, K.P.; Roest, H.P.; van der Laan, L.J.W.; Gietema, J.; Robert, J. Hamilton Looijenga Identification and Validation Model for Informative Liquid Biopsy-Based microRNA Biomarkers: Insights from Germ Cell Tumor In Vitro, in Vivo and Patient-Derived Data João. Cells 2019, 8, 1637. [CrossRef]

37. Peng, Y.; Croce, C.M. The role of microRNAs in human cancer. Signal Transduct. Target. Ther. 2016, 1, 15004. [CrossRef]

38. Agaoglu, F.Y.; Kovancilar, M.; Dizdar, Y.; Darendeliler, E.; Holdenrieder, S.; Dalay, N.; Gezer, U. Investigation of miR-21, miR-141, and miR-221 in blood circulation of patients with prostate cancer. Tumor Biol. 2011, 32, 583-588. [CrossRef]

39. Drabovich, A.P.; Saraon, P.; Jarvi, K.; Diamandis, E.P. Seminal plasma as a diagnostic fluid for male reproductive system disorders. Nat. Rev. Urol. 2014, 11, 278-288. [CrossRef]

40. Eskra, J.N.; Rabizadeh, D.; Pavlovich, C.P.; Catalona, W.J.; Luo, J. Approaches to urinary detection of prostate cancer. Prostate Cancer Prostatic Dis. 2019, 22, 362-381. [CrossRef]

41. Song, Q.; An, Q.; Niu, B.; Lu, X.; Zhang, N.; Cao, X. Role of miR-221/222 in Tumor Development and the Underlying Mechanism. J. Oncol. 2019, 2, 7252013. [CrossRef]

42. Mercatelli, N.; Coppola, V.; Bonci, D.; Miele, F.; Costantini, A.; Guadagnoli, M.; Bonanno, E.; Muto, G.; Frajese, G.V.; De Maria, R.; et al. The inhibition of the highly expressed mir-221 and mir-222 impairs the growth of prostate carcinoma xenografts in mice. PLoS ONE 2008, 3, e4029. [CrossRef] [PubMed] 
43. Zhou, C.F.; Ma, J.; Huang, L.; Yi, H.Y.; Zhang, Y.M.; Wu, X.G.; Yan, R.M.; Liang, L.; Zhong, M.; Yu, Y.H.; et al. Cervical squamous cell carcinoma-secreted exosomal miR-221-3p promotes lymphangiogenesis and lymphatic metastasis by targeting VASH1. Oncogene 2019, 38, 1256-1268. [CrossRef] [PubMed]

44. Pan, X.; Hong, X.; Lai, J.; Cheng, L.; Cheng, Y.; Yao, M.; Wang, R.; Hu, N. Exosomal MicroRNA-221-3p Confers Adriamycin Resistance in Breast Cancer Cells by Targeting PIK3R1. Front. Oncol. 2020, 10, 441. [CrossRef] [PubMed]

45. Ghosh, S.; Bhowmik, S.; Majumdar, S.; Goswami, A.; Chakraborty, J.; Gupta, S.; Aggarwal, S.; Ray, S.; Chatterjee, R.; Bhattacharyya, S.; et al. The exosome encapsulated microRNAs as circulating diagnostic marker for hepatocellular carcinoma with low alphafetoprotein. Int. J. Cancer 2020, 147, 2934-2947. [CrossRef]

46. Larrabeiti-Etxebarria, A.; Lopez-Santillan, M.; Santos-Zorrozua, B.; Lopez-Lopez, E.; Garcia-Orad, A. Systematic review of the potential of MicroRNAs in diffuse large B cell lymphoma. Cancers 2019, 11, 144. [CrossRef]

47. Wu, Y.; Wei, J.; Zhang, W.; Xie, M.; Wang, X.; Xu, J. Serum exosomal miR-1290 is a potential biomarker for lung adenocarcinoma. OncoTargets Ther. 2020, 13, 7809-7818. [CrossRef]

48. Kristensen, H.; Thomsen, A.R.; Haldrup, C.; Dyrskjøt, L.; Høyer, S.; Borre, M.; Mouritzen, P.; Ørntoft, T.F.; Sørensen, K.D. Novel diagnostic and prognostic classifiers for prostate cancer identified by genome-wide microRNA profiling. Oncotarget 2016, 7, 30760-30771. [CrossRef]

49. Kurul, N.O.; Ates, F.; Yilmaz, I.; Narli, G.; Yesildal, C.; Senkul, T. The association of let-7c, miR-21, miR-145, miR-182, and miR-221 with clinicopathologic parameters of prostate cancer in patients diagnosed with low-risk disease. Prostate 2019, 79, 1125-1132. [CrossRef]

50. Galardi, S.; Mercatelli, N.; Giorda, E.; Massalini, S.; Frajese, G.V.; Ciafrè, S.A.; Farace, M.G. miR-221 and miR-222 expression affects the proliferation potential of human prostate carcinoma cell lines by targeting p27Kip1. J. Biol. Chem. 2007, 282, 23716-23724. [CrossRef]

51. He, X.Y.; Tan, Z.L.; Mou, Q.; Liu, F.J.; Liu, S.; Yu, C.W.; Zhu, J.; Lv, L.Y.; Zhang, J.; Wang, S.; et al. microRNA-221 enhances MYCN via targeting nemo-like kinase and functions as an oncogene related to poor prognosis in neuroblastoma. Clin. Cancer Res. 2017, 23, 2905-2918. [CrossRef] [PubMed]

52. Huang, Y.; Yang, Y.; He, Y.; Li, J. The emerging role of Nemo-like kinase (NLK) in the regulation of cancers. Tumor Biol. 2015, 36, 9147-9152. [CrossRef] [PubMed]

53. Emami, K.H.; Brown, L.G.; Pitts, T.E.M.; Sun, X.; Vessella, R.L.; Corey, E. Nemo-like kinase induces apoptosis and inhibits androgen receptor signaling in prostate cancer cells. Prostate 2009, 69, 1481-1492. [CrossRef] [PubMed]

54. Kishore, S.; Jaskiewicz, L.; Burger, L.; Hausser, J.; Khorshid, M.; Zavolan, M. A quantitative analysis of CLIP methods for identifying binding sites of RNA-binding proteins. Nat. Methods 2011, 8, 559-567. [CrossRef]

55. Mullany, L.E.; Herrick, J.S.; Wolff, R.K.; Stevens, J.R.; Samowitz, W.; Slattery, M.L. Transcription factor-microRNA associations and their impact on colorectal cancer survival. Mol. Carcinog. 2017, 56, 2512-2526. [CrossRef]

56. Chen, W.S.; Chen, C.C.; Chen, L.L.; Lee, C.C.; Huang, T.S. Secreted heat shock protein $90 \alpha(H S P 90 \alpha)$ induces nuclear factor-kBmediated TCF12 protein expression to down-regulate E-cadherin and to enhance colorectal cancer cell migration and invasion. J. Biol. Chem. 2013, 288, 9001-9010. [CrossRef]

57. Chen, Q.B.; Liang, Y.K.; Zhang, Y.Q.; Jiang, M.Y.; Han, Z.D.; Liang, Y.X.; Wan, Y.P.; Yin, J.; He, H.C.; Zhong, W. De Decreased expression of TCF12 contributes to progression and predicts biochemical recurrence in patients with prostate cancer. Tumor Biol. 2017, 39. [CrossRef]

58. Zhang, M.; Xiao, X.; Xiong, D.; Liu, Q. Accurate normalization of real-time quantitative RT-PCR data by geometric averaging of multiple internal control genes. J. Artif. Intell. Res. 2014, 50, 1-30. [CrossRef]

59. Vendrell, J.; Maymó-Masip, E.; Tinahones, F.; García-España, A.; Megia, A.; Caubet, E.; García-Fuentes, E.; Chacón, M.R. Tumor necrosis-like weak inducer of apoptosis as a proinflammatory cytokine in human adipocyte cells: Up-regulation in severe obesity is mediated by inflammation but not hypoxia. J. Clin. Endocrinol. Metab. 2010, 95, 2983-2992. [CrossRef]

60. Riffo-Campos, Á.L.; Riquelme, I.; Brebi-Mieville, P. Tools for sequence-based miRNA target prediction: What to choose? Int. J. Mol. Sci. 2016, 17, 1987. [CrossRef]

61. Willkomm, S.; Zander, A.; Grohmann, D. Drug Target miRNA. Methods Mol. Biol. 2017, 1517, 291-304. [CrossRef] [PubMed]

62. Sticht, C.; De La Torre, C.; Parveen, A.; Gretz, N. Mirwalk: An online resource for prediction of microrna binding sites. PLoS ONE 2018, 13, e0206239. [CrossRef] [PubMed] 